\title{
Continuous phase spaces and the time evolution of spins: star products and spin-weighted spherical harmonics
}

\author{
Bálint Koczor, Robert Zeier, and Steffen J. Glaser \\ Department Chemie, Technische Universität München, \\ Lichtenbergstrasse 4, 85747 Garching, Germany
}

\begin{abstract}
We study continuous phase spaces of single spins and develop a complete description of their time evolution. The time evolution is completely specified by so-called star products. We explicitly determine these star products for general spin numbers using a simplified approach which applies spin-weighted spherical harmonics. This approach naturally relates phase spaces of increasing spin number to their quantum-optical limit and allows for efficient approximations of the time evolution for large spin numbers. We also approximate phase-space representations of certain quantum states that are challenging to calculate for large spin numbers. All of these applications are explored in concrete examples and we outline extensions to coupled spin systems.
\end{abstract}

Keywords: phase-space methods, spin systems, time evolution, star product, spinweighted spherical harmonics

\section{Introduction}

Phase-space techniques provide a complete description of quantum mechanics which is complementary to Hilbert-space [1] and path-integral [2] methods. These techniques are widely used in order to describe, visualize, and analyze quantum states [3-12]. Particular cases include Wigner [13], Husimi Q [14], and Glauber P [15 functions. In this work, we are particularly interested in phase-space methods that are applicable to (finite-dimensional) spin systems [16-27] and how these methods are related to infinitedimensional phase spaces [28]. Building on earlier results in [17, 18, 21, 22, 27, 29], we have developed in [28] a unified description for the general class of $s$-parametrized phase spaces with $-1 \leq s \leq 1$ which is applicable to single spins with integer or half-integer spin number $J$ and which naturally recovers the infinite-dimensional case in the large$J$ limit. The $s$-parametrized phase-space function corresponding to a Hilbert-space operator $A$ is denoted by $F_{A}(\Omega, s)$.

A new focus emerged recently with the objective to faithfully describe coupled spin systems with the help of phase-space representations [29 38] while also emphasizing 


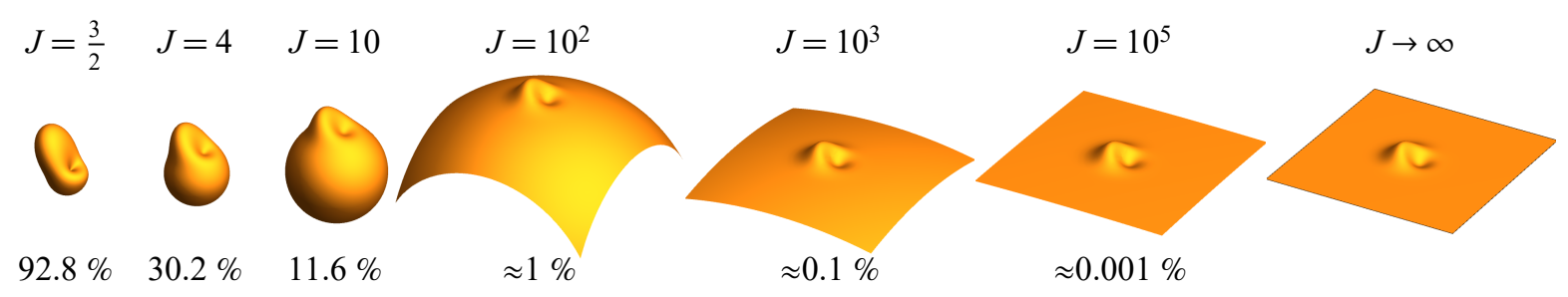

Figure 1. Example of how methods developed in this work can be applied (see section 10): Efficiently computed approximations of spherical Wigner functions of the excited spin coherent state $\left|\Omega_{0}^{+}\right\rangle$(see $\left[69\right.$ ) are shown together with their $L^{2}$ errors. They concentrate at the north pole for increasing spin number $J$ and approach the photon-added coherent state $\left|\alpha_{0}^{+}\right\rangle$(see (64)), as the spherical phase space converges to the planar one. The spherical Wigner functions are plotted on a sphere of radius $R:=\sqrt{J /(2 \pi)}$.

their spin-local properties. In this context, we have completely characterized the time evolution of Wigner functions for coupled spins $1 / 2$ in [34 using explicit star products 19, 23, 34. Star products are an important concept in the phase-space description of the time evolution and they determine the phase-space function

$$
F_{A B}(\Omega, s)=F_{A}(\Omega, s) \star^{(s)} F_{B}(\Omega, s),
$$

of a product of two Hilbert-space operators $A B$ in terms of the individual phase-space functions $F_{A}(\Omega, s)$ and $F_{B}(\Omega, s)[34]$. This results in the so-called Moyal equation

$$
\frac{\partial F_{\rho}(\Omega, s)}{\partial t}=-i F_{\mathcal{H}}(\Omega, s) \star^{(s)} F_{\rho}(\Omega, s)+i F_{\rho}(\Omega, s) \star^{(s)} F_{\mathcal{H}}(\Omega, s)
$$

which describes time evolution of a quantum state $F_{\rho}(\Omega, s)$ under a Hamiltonian $F_{\mathcal{H}}(\Omega, s)$ directly in phase-space (cf. 34]).

In this work, we extend our earlier results in [34] on Wigner functions of coupled spins $1 / 2$ and present the explicit form of the star product for the general class of $s$ parametrized phase spaces which is applicable to single and coupled spins of arbitrary spin number $J$. We also rely on phase-space techniques for single spins $J$ that have been developed in [28]. We introduce spin-weighted spherical harmonics 39] as an important new technical tool to the theory of phase spaces, even though they have not been considered in this context before. This allows us to significantly simplify the theory of phase spaces and their star products. In particular, we can now efficiently approximate the time evolution of phase-space representations for single spins that have a large spin number $J$. Many quantum states have quite complicated phase-space representations which are challenging to calculate for large values of $J$. Approximation methods for the time evolution also lead to efficient computational techniques for approximating phasespace representations of spins with large $J$ and figure 1 illustrates this limit for Wigner functions of excited spin coherent states. Relying on results from [34], we outline in the main text how our results can be also extended to coupled spin systems.

Let us compare our work with earlier results. The star product of Husimi Q functions of single spins has been derived in [40] using angular-momentum operators. 
This approach has been independently rediscovered in 23, 24 and was used to calculate the star product and time evolution of Glauber $\mathrm{P}$ functions, and this has also been translated to the general case of $s$-parametrized phase-space representations. A semiclassical equation of motion was derived in 23 by neglecting quantum terms of the star product, which has then been applied to the semiclassical simulation of quantum dynamics 25] and the classical limit of spin Bopp operators [41. In contrast to this approach relying on angular momentum operators, our techniques based on spinweighted spherical harmonics and spin-weight raising and lowering operators facilitate a simplified and more systematic approach and also lead to additional formulas for the star product. The derivation of the exact star product is now completely transparent and all of its quantum contributions are accounted for. One particular strength of our approach is that the large-spin limit is naturally incorporated as spin-weight raising and lowering operators converge for large $J$ to derivatives in the tangent plane (which are widely studied in infinite dimensions [42]).

This work has the following structure: We start in section 2 by recapitulating elementary properties of infinite-dimensional phase spaces and their star products. In section 3, we recall the structure of phase-space representations for single spins $J$ following the approach of [28]. We introduce spin-weighted spherical harmonics and summarize their main features in section 4. Important approximation formulas for spin-weight raising and lowering operators are derived in section 5. The sections 6,8 constitute the main part of our work and various formulas for exact and approximate star products are obtained. Our methods are illustrated with concrete examples in sections 9, 10. Before we conclude, extension to coupled spin systems are outlined in section 11. Certain details and proofs are deferred to appendices.

\section{Phase spaces and star products in infinite dimensions}

An important class of infinite-dimensional phase-space representations of a density operator $\rho$ contains $s$-parametrized phase-space distribution functions (where $-1 \leq$ $s \leq 1)$ which can be defined via $3,15,28,43,44$

$$
F_{\rho}(\Omega, s)=\operatorname{Tr}\left[\rho \mathcal{D}(\Omega) \Pi_{s} \mathcal{D}^{\dagger}(\Omega)\right]
$$

The distribution function $F_{\rho}(\Omega, s)$ is determined by the expectation value of the parity operator $\Pi_{s}$ which is transformed by the displacement operator $\mathcal{D}(\Omega)$. The parity operator inverts phase-space coordinates via $\Pi_{0}|\Omega\rangle=|-\Omega\rangle, 44$ and the displacement operator $\mathcal{D}(\Omega)$ is defined by the property that it translates the vacuum state $|0\rangle$ to coherent states $\mathcal{D}(\Omega)|0\rangle=|\Omega\rangle$. Here, $\Omega$ parametrizes a phase space with either the variables $p$ and $q$ or the complex eigenvalues $\alpha$ of the annihilation operator $[3]$. And the parameter $s$ interpolates between the Glauber P function for $s=1$ and the Husimi $\mathrm{Q}$ function for $s=-1$. The particular case of $s=0$ corresponds to the Wigner function. All $s$-parametrized phase-space distribution functions are related to each other via Gaussian smoothing [15, 28], and the convolution of the vacuum-state representation 
$F_{|0\rangle}\left(\Omega, s^{\prime}\right)$ with the distribution function $F_{\rho}(\Omega, s)$ results in a distribution function

$$
F_{\rho}\left(\Omega, s+s^{\prime}-1\right)=F_{|0\rangle}\left(\Omega, s^{\prime}\right) * F_{\rho}(\Omega, s)=\exp \left[\frac{1-s^{\prime}}{2} \partial_{\alpha^{*}} \partial_{\alpha}\right] F_{\rho}(\Omega, s)
$$

of type $s+s^{\prime}-1$. The r.h.s. of (4) establishes the corresponding differential form, see, e.g., Eq. (5.29) in 45].

We adapt notations from [21] for the infinite-dimensional tensor operators $\mathrm{T}_{\nu}:=$ $\mathcal{D}(\nu)$ which define the displacement operators using a continuous, complex index $\nu$. The phase-space representations $F_{\mathrm{T}_{\nu}}(\alpha, s)=\gamma_{\nu}^{-s} \mathrm{Y}_{\nu}(\alpha)$ are up to the weight factor $\gamma_{\nu}:=\exp \left(-|\nu|^{2} / 2\right)$ proportional to the harmonic functions $\mathrm{Y}_{\nu}(\alpha):=\exp \left(\nu \alpha^{*}-\alpha \nu^{*}\right)$ 21], where the power $-s$ of $\gamma_{\nu}$ is determined by the type $s$ of the representation. Up to a complex prefactor, multiplying two displacement operators results in a single displacement operator [15]:

$$
\mathrm{T}_{\mu} \mathrm{T}_{\nu}=\exp \left[\left(\mu \nu^{*}-\nu \mu^{*}\right) / 2\right] \mathrm{T}_{\mu+\nu} .
$$

Applying the product in (5) and the star product from (1), one obtains the formula

$$
\left[\gamma_{\mu}^{-s} \mathrm{Y}_{\mu}(\alpha)\right] \star^{(s)}\left[\gamma_{\nu}^{-s} \mathrm{Y}_{\nu}(\alpha)\right]=\exp \left[\left(\mu \nu^{*}-\nu \mu^{*}\right) / 2\right] \gamma_{\mu+\nu}^{-s} \mathrm{Y}_{\mu+\nu}(\alpha)
$$

The star product satisfies (6) and it can be explicitly defined as a power series

$$
\star^{(s)}:=\exp \left[\frac{(1-s)}{2} \overleftarrow{\partial}_{\alpha} \vec{\partial}_{\alpha^{*}}-\frac{(1+s)}{2} \overleftarrow{\partial}_{\alpha^{*}} \vec{\partial}_{\alpha}\right]
$$

of partial derivatives as in Eq. (3.5) of $\left[42\right.$. Setting $\alpha=\left(\lambda q+i \lambda^{-1} p\right) / \sqrt{2}$ for arbitrary real $\lambda$ [15], the derivatives observe (see also Eq. (3.4') in [42])

$$
\frac{(1-s)}{2} \overleftarrow{\partial}_{\alpha} \vec{\partial}_{\alpha^{*}}-\frac{(1+s)}{2} \overleftarrow{\partial}_{\alpha^{*}} \vec{\partial}_{\alpha}=i\left[\overleftarrow{\partial}_{q} \vec{\partial}_{p}-\overleftarrow{\partial}_{p} \vec{\partial}_{q}-s \lambda^{2} \overleftarrow{\partial}_{p} \vec{\partial}_{p}-s \lambda^{-2} \overleftarrow{\partial}_{q} \vec{\partial}_{q}\right] / 2
$$

The arrows represent whether derivatives are to be taken to the left or right. For $s=0$, we obtain $\star^{(0)}=\exp (i\{\cdot, \cdot\} / 2)$ as stated by Groenewold $[46]$ and $\{\cdot, \cdot\}=\overleftarrow{\partial}_{q} \vec{\partial}_{p}-\overleftarrow{\partial}_{p} \vec{\partial}_{q}$ denotes the Poisson bracket.

\section{Finite-dimensional phase spaces}

We briefly review s-parametrized phase-space representations for a single spin with spin number $J$ following the approach of [28], which recovers the previously discussed infinite-dimensional case in the large-spin limit. The continuous phase space for the finite-dimensional spin $J$ is fully parametrized using the two Euler angles $\Omega:=(\theta, \phi)$ of the rotation operator $\mathcal{R}(\Omega)=\mathcal{R}(\theta, \phi):=e^{i \phi \mathcal{J}_{z}} e^{i \theta \mathcal{J}_{y}}$. Here, $\mathcal{J}_{z}$ and $\mathcal{J}_{y}$ are components of the angular momentum operator that are defined by their commutation relations, i.e., $\left[\mathcal{J}_{j}, \mathcal{J}_{k}\right]=i \sum_{\ell} \epsilon_{j k \ell} \mathcal{J}_{\ell}$ where $j, k, \ell \in\{x, y, z\}$ and $\epsilon_{j k \ell}$ is the Levi-Civita symbol [47. This leads to a spherical phase space with radius $R:=\sqrt{J /(2 \pi)}$. The displacement operator $\mathcal{D}(\Omega)$ from the infinite-dimensional case is replaced by the rotation operator $\mathcal{R}(\Omega)$ which maps the spin-up state $|J J\rangle$ to spin coherent states $|\Omega\rangle=\mathcal{R}(\Omega)|J J\rangle$ 18, 48, 49. The $s$-parametrized phase-space representation

$$
F_{\rho}(\Omega, s):=\operatorname{Tr}\left[\rho \mathcal{R}(\Omega) M_{s} \mathcal{R}^{\dagger}(\Omega)\right]
$$


of a density operator $\rho$ of a single spin $J$ is then obtained as the expectation value of the rotated (generalized) parity operator (refer to [28])

$$
M_{s}:=\frac{1}{R} \sum_{j=0}^{2 J} \sqrt{\frac{2 j+1}{4 \pi}}\left(\gamma_{j}\right)^{-s} \mathrm{~T}_{j 0}
$$

where $M_{s}$ is specified in terms of a weighted sum of tensor operators $\mathrm{T}_{j m}$ of order zero (i.e., $m=0$ ). The tensor components $\mathrm{T}_{j m}$ depend on the rank $j \in\{0, \ldots, 2 J\}$, the order $m \in\{-j, \ldots, j\}$, and the spin number $J$. The explicit matrix elements are specified in terms of Clebsch-Gordan coefficients [21, 47, 50, 51] as

$$
\left[\mathrm{T}_{j m}\right]_{m_{1} m_{2}}:=\sqrt{\frac{2 j+1}{2 J+1}} C_{J m_{2}, j m}^{J m_{1}}=(-1)^{J-m_{2}} C_{J m_{1} J,-m_{2}}^{j m},
$$

where $m_{1}, m_{2} \in\{J, \ldots,-J\}$. The weight factor has the explicit form

$$
\gamma_{j}:=R \sqrt{4 \pi}(2 J) ![(2 J+j+1) !(2 J-j) !]^{-1 / 2},
$$

and the power $-s$ of $\gamma_{j}$ determines the type $s$ of the phase-space representation [28].

Tensor operators $\mathrm{T}_{j m}$ form an orthonormal basis of $(2 J+1) \times(2 J+1)$ matrices with respect to the Hilbert-Schmidt scalar product $\operatorname{Tr}\left[\mathrm{T}_{j m} \mathrm{~T}_{j^{\prime} m^{\prime}}^{\dagger}\right]=\delta_{j j^{\prime}} \delta_{m m^{\prime}}$ where $0 \leq j \leq 2 J$ and $m, m^{\prime} \in\{-j, \ldots, j\}$. Similarly as in (5), the product of two tensor operators can be decomposed into a sum (applying the notation of [34])

$$
\mathrm{T}_{j m} \mathrm{~T}_{j^{\prime} m^{\prime}}=\sum_{\ell=0}^{2 J} K_{j m, j^{\prime} m^{\prime}}^{\ell} \mathrm{T}_{\ell, m+m^{\prime}}
$$

of tensor operators using the decomposition coefficients $K_{j m, j^{\prime} m^{\prime}}^{\ell}$ as detailed in Appendix A. The phase-space representations $F_{\mathrm{T}_{j m}}(\Omega, s)=\gamma_{j}^{-s} \mathrm{Y}_{j m}(\Omega) / R$ of tensor operators are proportional to spherical harmonics of rank $j$ and order $m$ and they are orthonormal with respect to a spherical integration

$$
\int_{S^{2}} \mathrm{Y}_{j m}(\Omega) \mathrm{Y}_{j^{\prime} m^{\prime}}^{*}(\Omega) / R^{2} \mathrm{~d} \Omega=\delta_{j j^{\prime}} \delta_{m m^{\prime}}
$$

according to $\mathrm{d} \Omega=R^{2} \sin \theta \mathrm{d} \theta \mathrm{d} \phi$. Similarly as in (6), the defining property of the star product for phase-space representations of spins can be transferred to spherical harmonics since these distribution functions are always given as a finite linear combination of spherical harmonics:

Definition 1. As in (1), the star product $\star^{(s)}$ of two phase-space representations of type $s$ satisfies for a single spin $J$ the condition

$$
\left[\gamma_{j}^{-s} \mathrm{Y}_{j m}(\Omega)\right] \star^{(s)}\left[\gamma_{j^{\prime}}^{-s} \mathrm{Y}_{j^{\prime} m^{\prime}}(\Omega)\right]=R \sum_{\ell=0}^{2 J} K_{j m, j^{\prime} m^{\prime}}^{\ell} \gamma_{\ell}^{-s} \mathrm{Y}_{\ell, m+m^{\prime}}(\Omega)
$$

for all suitable indices with $j, j^{\prime} \leq 2 J$. The coefficients $K_{j m, j^{\prime} m^{\prime}}^{\ell}$ are determined by (12).

One objective of this work is to apply this decomposition in order to define a star product $\star^{(s)}$ in terms of spin-weighted spherical harmonics and their spin-weight raising and lowering differential operators $\bar{\partial}$ and $\bar{\partial}$. 


\section{Spin-weighted spherical harmonics}

Spin-weighted spherical harmonics $\mathrm{Y}_{j m}^{\eta}$ with spin weight $\eta$ have been introduced by Newman and Penrose [39] using spin-weight raising and lowering operators $\partial$ and $\bar{\partial}$ in order to describe the asymptotic behavior of the gravitational field. Since then, spin-weighted spherical harmonics have been widely used to analyze, in particular, gravitational waves [52, 53] or the cosmic microwave background [54,55]. Moreover, efficient computational tools for spin-weighted spherical harmonics are available [56] and these can also be used in the fast calculation of spherical convolutions [57, 58].

Spin-weighted spherical harmonics $\mathrm{Y}_{j m}^{\eta}$ with $-j \leq \eta \leq j$ are defined as functions on the three-dimensional sphere as shown in figure 2. Similarly as for ordinary spherical harmonics $\mathrm{Y}_{j m}(\theta, \phi)$, the spin-weight raising and lowering operators $\widetilde{\partial}$ and $\bar{\partial}$ are used in their definition (see, e.g., 39] and Chapter 2.3 in [59])

$$
\mathrm{Y}_{j m}^{\eta}:=\left\{\begin{array}{cc}
\sqrt{(j-\eta) ! /(j+\eta) !} \delta^{\eta} \mathrm{Y}_{j m} & \text { for } \eta \geq 0 \\
(-1)^{\eta} \sqrt{(j-|\eta|) ! /(j+|\eta|) !} \bar{\delta}^{|\eta|} \mathrm{Y}_{j m} & \text { for } \eta<0,
\end{array}\right.
$$

and the particular case of $\eta=0$ corresponds to ordinary spherical harmonics. (Spinweighted spherical harmonics are related to Wigner D-matrices via $D_{m \eta}^{j}(\phi, \theta, \psi)=$ $(-1)^{m} \sqrt{(4 \pi) /(2 j+1)} Y_{j,-m}^{\eta}(\theta, \phi) e^{-i \eta \psi}$, refer to Eq. (2.52) in $[59 \mid$.) The operators $\partial$ and $\bar{\partial}$ raise and lower the spin weight $\eta$ with $-j \leq \eta \leq j$ in (see Eq. (3.20) in [39])

$$
\partial Y_{j m}^{\eta}=\sqrt{(j-\eta)(j+\eta+1)} Y_{j m}^{\eta+1} \text { and } \bar{\partial} Y_{j m}^{\eta}=-\sqrt{(j+\eta)(j-\eta+1)} Y_{j m}^{\eta-1} .
$$

Their explicit form can be specified in terms of the differential operators

$$
\begin{aligned}
& \partial Y_{j m}^{\eta}=-(\sin \theta)^{\eta}\left(\partial_{\theta}+i / \sin \theta \partial_{\phi}\right)\left[(\sin \theta)^{-\eta} Y_{j m}^{\eta}\right] \text { and } \\
& \bar{\partial} Y_{j m}^{\eta}=-(\sin \theta)^{-\eta}\left(\partial_{\theta}-i / \sin \theta \partial_{\phi}\right)\left[(\sin \theta)^{\eta} Y_{j m}^{\eta}\right],
\end{aligned}
$$

see Eq. (3.8) in [39]. Spin-weighted spherical harmonics are up to a constant factor invariant under the application of $ð \bar{\partial}$ and $\bar{\varnothing} \partial$ (see Eq. (2.22) in [59]):

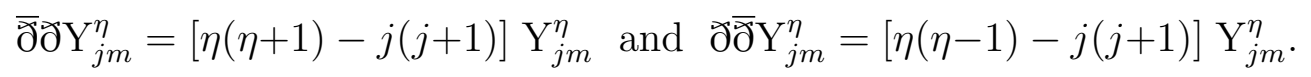

Therefore, $\partial \bar{\partial}$ acts up to a minus sign as the total angular momentum operator when applied to spherical harmonics, i.e., $\bar{\varnothing} \bar{\gamma} \mathrm{Y}_{j m}=-j(j+1) \mathrm{Y}_{j m}$, refer to Eq. (2.25) in [59]. The commutator $[\bar{\partial}, \check{\partial}]=2 \eta$ immediately follows from $\left[19 p\right.$. Products $Y_{j m}^{\eta} Y_{j^{\prime} m^{\prime}}^{-\eta} \propto$ $\left(\widetilde{\partial}^{\eta} Y_{j m}\right)\left(\bar{\partial}^{\eta} Y_{j m}\right)$ of spin-weighted spherical harmonics decompose into the sums [59]

$$
\begin{aligned}
& \left(\check{\partial}^{\eta} Y_{j m}\right)\left(\bar{\partial}^{\eta} Y_{j^{\prime} m^{\prime}}\right)=\sum_{\ell=0}^{j+j^{\prime}}{ }^{\eta} \kappa_{j m, j^{\prime} m^{\prime}}^{\ell} \mathrm{Y}_{\ell, m+m^{\prime}}(\Omega) \text { and } \\
& \left(\bar{\delta}^{\eta} \mathrm{Y}_{j m}\right)\left(\check{\partial}^{\eta} \mathrm{Y}_{j^{\prime} m^{\prime}}\right)=\sum_{\ell=0}^{j+j^{\prime}}{ }^{-\eta} \kappa_{j m, j^{\prime} m^{\prime}}^{\ell} \mathrm{Y}_{\ell, m+m^{\prime}}(\Omega)
\end{aligned}
$$

of spherical harmonics. The decomposition coefficients ${ }^{\eta} \kappa_{j m, j^{\prime} m^{\prime}}^{\ell}$ are explicitly specified in A.5 and they are similar to the ones used in Definition 1. In sections 648, we utilize the products of spin-weighted spherical harmonics from (20) and (21) to explicitly determine the star product such that it satisfies its defining property from (1). 

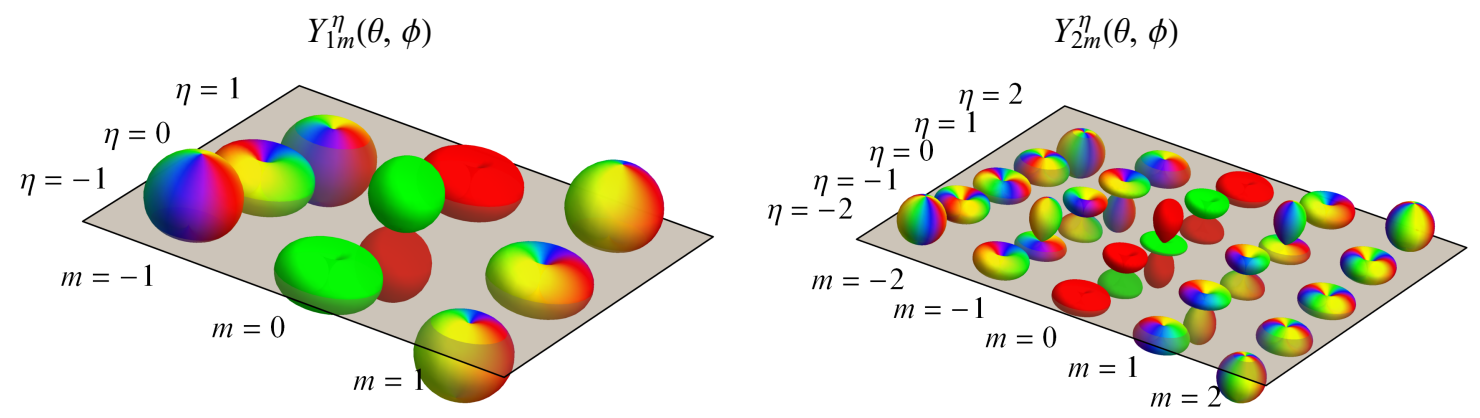

Figure 2. Spin-weighted spherical harmonics $\mathrm{Y}_{j m}^{\eta}$ of rank one (left) and two (right). Colors represent the complex phase; red (dark gray) and green (light gray) depict positive and negative values, while blue and yellow represent $i$ and $-i$.

\section{Approximating spin-weight raising and lowering operators}

As mentioned in section 3, the spherical phase space converges for an increasing spin number $J$ to the (infinite-dimensional) planar phase space [28]. The arc length $\theta R$ becomes a measure of distance from the north pole, which is equivalent to its infinitedimensional counterpart $|\alpha|$. The two phase spaces can be related using the formula $\alpha=\sqrt{J / 2} \theta e^{-i \phi}$. In this parametrization, spin-weighted spherical harmonics can, up to an additive error that scales inversely with $R$, be expanded as derivatives

$$
\begin{aligned}
\mathrm{Y}_{j m}^{\eta}(\alpha) & =(-1)^{\eta} e^{-i \eta \phi}\left(\partial_{\alpha^{*}}\right)^{\eta} \mathrm{Y}_{j m}(\alpha)+\mathcal{O}(|\alpha| / \sqrt{J}) \text { and } \\
\mathrm{Y}_{j m}^{-\eta}(\alpha) & =e^{i \eta \phi}\left(\partial_{\alpha}\right)^{\eta} \mathrm{Y}_{j m}(\alpha)+\mathcal{O}(|\alpha| / \sqrt{J})
\end{aligned}
$$

of ordinary spherical harmonics with respect to the coordinates $\alpha$ and $\alpha^{*}$ while assuming a fixed arc length $|\alpha|$. This is essentially an approximation of (15) for small angles $\theta$. Figure 3(a)-(b) plots the absolute value of the difference between the spin-weighted spherical harmonics $\mathrm{Y}_{j m}^{\eta}(\alpha)$ and their approximations which rely on the derivatives from (22)- (23). The approximation error vanishes in the limit of large $J$ assuming that the coordinates $\alpha$ are located at the north pole or that the values $|\alpha| / \sqrt{J}$ are small (e.g., $|\alpha|$ is bounded). Extending this to the spin-weight raising and lowering operators from (17) and (18), these operators $\widetilde{\partial}$ and $\bar{\partial}$ can be shown to transform for large $J$ into the derivatives $\partial_{\alpha^{*}}$ and $\partial_{\alpha}$ over the complex plane.

Proposition 1. Assume that the phase-space function $f=F_{\rho}(\Omega, s)$ of a spin $J$ is parametrized using the arc length $\alpha=\sqrt{J / 2} \theta e^{-i \phi}$ and that its spherical-harmonics expansion coefficients might depend on $J$. The action of spin-weight raising and lowering operators $\check{\partial}$ and $\overline{\widetilde{\partial}}$ at fixed $\alpha$ are given by

$$
\begin{aligned}
& {\left[(ð / \sqrt{2 J})^{\eta} f\right](\alpha)=(-1)^{\eta} e^{-i \eta \phi}\left(\partial_{\alpha^{*}}\right)^{\eta} f(\alpha)+\mathcal{O}\left(|\alpha| J^{-1}\right),} \\
& {\left[(\overline{\mathrm{\partial}} / \sqrt{2 J})^{\eta} f\right](\alpha)=(-1)^{\eta} e^{i \eta \phi}\left(\partial_{\alpha}\right)^{\eta} f(\alpha)+\mathcal{O}\left(|\alpha| J^{-1}\right),} \\
& {[(ð \bar{\delta} /(2 J)) f](\alpha)=\partial_{\alpha^{*}} \partial_{\alpha} f(\alpha)+\mathcal{O}\left(|\alpha| J^{-1}\right) \text {, and }} \\
& {[(\overline{\widetilde{\partial}} /(2 J)) f](\alpha)=\partial_{\alpha} \partial_{\alpha^{*}} f(\alpha)+\mathcal{O}\left(|\alpha| J^{-1}\right),}
\end{aligned}
$$




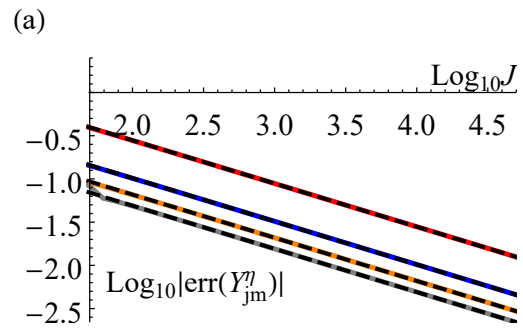

(b)

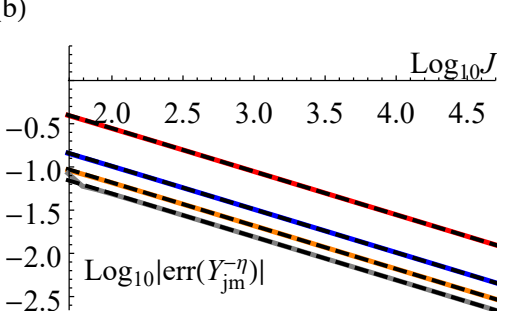

(c)

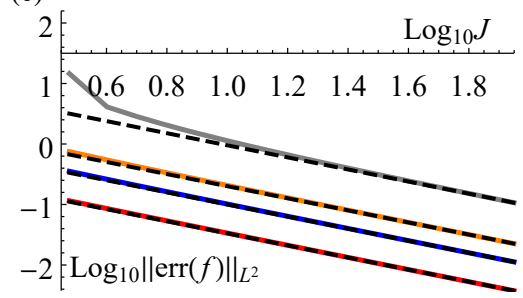

Figure 3. The absolute value of the difference between exact spin-weighted spherical harmonics (a) $\mathrm{Y}_{j m}^{\eta}(\alpha)$ and $(\mathrm{b}) \mathrm{Y}_{j m}^{-\eta}(\alpha)$ and their approximations from 22 and 23 for fixed $\eta=4, m=4$, and $\alpha=1.2 e^{i 2.1}$ and variable $j \in\{4,8,12,16\}$ (red, blue, orange, gray). (c) $L^{2}$-norm of the difference between the exact derivative of the spin-up state Wigner function $f:=[\overline{\widetilde{\partial}} / \sqrt{2 J}]^{n} W_{|J J\rangle}(\Omega)$ and its approximation using Proposition 1 for $n \in\{2,3,4,8\}$ (red, blue, orange, gray). Dashed lines show the expected scaling $\mathcal{O}\left(J^{-1 / 2}\right)$ for (a) and (b) as well as $\mathcal{O}\left(J^{-1}\right)$ for (c) with suitable prefactors.

and this action is up to an error term $\mathcal{O}\left(J^{-1}\right)$ equivalent to applying the complex derivatives $\partial_{\alpha^{*}}$ and $\partial_{\alpha}$ for any powers of $\eta$. The error term $\mathcal{O}\left(J^{-1}\right)$ vanishes in the limit of large $J$ if the differentials $[ð / \sqrt{2 J}]^{\eta} f$ remain non-singular in the limit. This implies convergence in the $L^{2}$ norm if $f$ and its differentials are also square integrable in the limit. Refer to Appendix E for the proof.

Note that phase-space representations $F_{\rho}(\Omega, s)$ and all their derivatives are nonsingular and square integrable if $\rho$ is finite-dimensional, i.e., if $F_{\rho}(\Omega, s)$ is a finite linear combination of spherical harmonics. In general, singularities can however appear for $s>0$ in the limit of large $J$, as the corresponding parity operators $\Pi_{s}$ from (3) are unbounded 15,28 for $s>0$. This can be illustrated using the example of the spin-up state $F_{|J J\rangle}(\Omega, s)$ : it is determined by a sum of $2 J+1$ spherical harmonics and its expansion coefficients are proportional to $\gamma_{j}^{1-s}$ and depend implicitly on $J$ [28]. These rapidly decreasing expansion coefficients can be approximated for increasing $j$ by $e^{-j^{2}(1-s) /(4 J)}$ if $s<1$ and $F_{|J J\rangle}(\Omega, s)$ is bounded and square integrable in the large- $J$ limit. But for $s=1$ this expansion defines in the limit a delta distribution which is clearly singular and not square integrable. For example, the differentials $[\overline{\widetilde{\partial}} / \sqrt{2 J}]^{\eta} F_{|J J\rangle}(\Omega, s)$ are sums of spin-weighted spherical harmonics with expansion coefficients which are proportional to $[(2 J)(j-\eta) ! /(j+\eta) !]^{-\eta / 2} \gamma_{j}^{1-s}$ and which can be approximated by $\left[j^{2} /(2 J)\right]^{\eta / 2} e^{-j^{2}(1-s) /(4 J)}$. The coefficients vanish for increasing $j$ and define bounded, square-integrable functions in the limit of large $J$ for $s<1$. Figure 3(c) shows the $L^{2}$ norm of the difference between the Wigner function's differential $[\overline{\mathrm{d}} / \sqrt{2 J}]^{\eta} W_{|J J\rangle}(\Omega)$ and its approximation via Proposition 1. This difference vanishes for large $J$. Refer to Appendix E for further details.

One example of an unbounded operator is the Wigner function $W_{\mathcal{I}^{+} / \sqrt{2 J}}(\Omega)$ of the raising operator $\mathcal{I}^{+} / \sqrt{2 J}$ which reproduces the annihilation operator $a$ in the large-spin limit $[49]$. One obtains

$$
W_{\mathcal{I}^{+} / \sqrt{2 J}}(\Omega) \propto \sqrt{J / 2} \mathrm{Y}_{1,1}(\theta, \phi) \propto \sqrt{J / 2} \sin \theta e^{i \phi} \text { and }
$$


Continuous phase spaces and the time evolution of spins

$$
\bar{\delta} / \sqrt{2 J} W_{\mathcal{I}^{+} / \sqrt{2 J}}(\Omega) \propto \frac{1}{2} Y_{1,1}^{-1}(\theta, \phi) \propto \frac{1}{2}(1+\cos \theta) e^{i \phi} .
$$

The corresponding $L^{2}$ norms (as defined with respect to 13 ) diverge with increasing $J$ and the Wigner functions are no longer square integrable. However, for any bounded $\alpha=\sqrt{J / 2} \theta e^{-i \phi}$, the functions have the proper limits

$$
\lim _{J \rightarrow \infty}\left[\sqrt{J / 2} \sin (|\alpha| / \sqrt{J / 2}) e^{i \phi}\right]=\alpha \text { and } \lim _{J \rightarrow \infty}\left[\frac{1}{2}(1+\cos (|\alpha| / \sqrt{J / 2})]=1,\right.
$$

where 1 is the derivative of $\alpha$. Refer to Appendix E for details.

Proposition 1 essentially approximates the differentials $\varpi^{\eta} F_{\rho}(\Omega, s)$ of spherical functions with the derivatives $\partial_{\alpha^{*}} F_{\rho}(\Omega, s)$. This duality then becomes exact in the large-spin limit if the differentials $\varpi^{\eta} F_{\rho}(\Omega, s)$ remain non-singular. In particular, we use the spin-weight raising and lowering operators to construct the star product by applying (14), which then naturally recovers the infinite-dimensional star product from (7):

Proposition 2. Consider the arc-length parametrization $\alpha=\sqrt{J / 2} \theta e^{-i \phi}$ and two spin$J$ phase-space functions $f=F_{\rho}(\Omega, s)$ and $g=F_{\rho^{\prime}}(\Omega, s)$. Their spherical-harmonics expansion coefficients might depend on J. Following Proposition 1, one obtains the approximations

$$
\begin{aligned}
& f\left[\left(\overleftarrow{\partial}^{\eta}\right)\left(\overrightarrow{\bar{\partial}^{\eta}}\right) /(2 J)^{\eta}\right] g=f\left[\left(\overleftarrow{\partial}_{\alpha^{*}}\right)^{\eta}\left(\vec{\partial}_{\alpha}\right)^{\eta}\right] g+\mathcal{O}\left(J^{-1}\right) \\
& f\left[\left(\overleftarrow{\bar{\partial}^{\eta}}\right)\left(\overrightarrow{\partial^{\eta}}\right) /(2 J)^{\eta}\right] g=f\left[\left(\overleftarrow{\partial}_{\alpha}\right)^{\eta}\left(\vec{\partial}_{\alpha^{*}}\right)^{\eta}\right] g+\mathcal{O}\left(J^{-1}\right)
\end{aligned}
$$

The error vanishes in the limit of large $J$ if the differentials $\left[\check{\partial}^{\eta} f\right]\left[\bar{\partial}^{\eta} g\right] /(2 J)^{\eta}$ remain non-singular in the limit.

\section{Star products of spin Glauber P and Husimi Q functions}

\subsection{The exact star product}

We start by determining the exact star product of $\mathrm{Q}$ functions $(s=-1)$ and $\mathrm{P}$ functions $(s=1)$ which are given uniquely in terms of spin-weight raising and lowering operators:

Result 1. The (finite-dimensional) exact star product of two $Q$ functions $Q_{A}$ and $Q_{B}$ and two $P$ functions $P_{A}$ and $P_{B}$ is determined for the spin number $J$ by

$$
Q_{A} \star^{(-1)} Q_{B}=Q_{A} \sum_{\eta=0}^{2 J} \lambda_{\eta}^{(-1)}\left(\overleftarrow{\bar{ळ}^{\eta}}\right)\left(\overrightarrow{\check{\partial}^{\eta}}\right) Q_{B} \text { and } P_{A} \star^{(1)} P_{B}=P_{A} \sum_{\eta=0}^{2 J} \lambda_{\eta}^{(1)}\left(\overleftarrow{\check{\delta}^{\eta}}\right)\left(\overrightarrow{\bar{\sigma}^{\eta}}\right) P_{B}
$$

where the coefficients (see Appendix B)

$$
\lambda_{\eta}^{(-1)}=\frac{(2 J-\eta) !}{\eta !(2 J) !} \text { and } \lambda_{\eta}^{(1)}=\frac{R^{2} 4 \pi(-1)^{\eta}(2 J) !}{\eta !(2 J+\eta+1) !}
$$

depend on $J$. Terms in (32) related to spherical harmonics $\mathrm{Y}_{j m}$ with rank $j>2 J$ are not relevant and can be projected out as detailed in Appendix D and [34]. 
The proof of Result 1 is given in Appendix B. The upper bounds in the sums in 32 can be lowered to $\min \left(j_{A}, j_{B}\right)$ where $j_{A}$ and $j_{B}$ are the maximal ranks in the tensoroperator decompositions of $A$ and $B$. Similar results have been attained for $\mathrm{Q}$ functions in Eq. (86) of [40] using angular momentum operators, refer also to Eq. (45) in [23]. We improve and simplify these results for star products of phase-space representations of spins with the help of spin-weighted spherical harmonics. In particular, this approach enables us to efficiently approximate phase-space representations for large spin numbers $J$ as discussed below.

\subsection{Approximations of the star product}

The coefficients in (33) of Result 1 can be expanded as (see Appendix C)

$$
\begin{aligned}
& \lambda_{\eta}^{(-1)}=\left[\eta !(2 J)^{\eta}\right]^{-1} \text { for } \eta=0 \text { or } \eta=1, \\
& \lambda_{\eta}^{(-1)}=\left[\eta !(2 J)^{\eta}\right]^{-1}+\mathcal{O}\left(J^{-\eta-1}\right) \text { for } \eta \geq 2, \text { and } \\
& \lambda_{\eta}^{(1)}=(-1)^{\eta}\left[\eta !(2 J)^{\eta}\right]^{-1}+\mathcal{O}\left(J^{-\eta-1}\right) \text { for } \eta \geq 0 .
\end{aligned}
$$

Also, the finite sums in (32) are unchanged if higher-order differentials (with respect to $\eta$ ) are added since all the higher-order differentials vanish, i.e., $\partial^{\eta} Y_{j m}=0$ for $\eta>j$. The scaling $\mathcal{O}\left(J^{-\eta-1}\right)$ of the approximations in $(35)$ and $(36)$ is highlighted in figure $4(\mathrm{a})$-(b) for different values of $\eta$.

Result 2. The exact star product in Result 1 can be approximated as

$$
\begin{aligned}
& Q_{A} \star^{(-1)} Q_{B}=Q_{A} \exp [(\overleftarrow{\overline{\bar{\varpi}}})(\overrightarrow{\check{\partial}}) /(2 J)] Q_{B}+\mathcal{O}\left(J^{-3}\right) \\
& P_{A} \star^{(1)} P_{B}=P_{A} \exp [-(\overleftarrow{\check{\check{\partial}}})(\overrightarrow{\overline{\check{\partial}}}) /(2 J)] P_{B}+\mathcal{O}\left(J^{-1}\right) .
\end{aligned}
$$

One obtains from Proposition 1 a more convenient approximation

$$
\begin{aligned}
& Q_{A} \star^{(-1)} Q_{B}=Q_{A} \exp \left[\overleftarrow{\partial}_{\alpha} \vec{\partial}_{\alpha^{*}}\right] Q_{B}+\mathcal{O}\left(J^{-1}\right) \\
& P_{A} \star^{(1)} P_{B}=P_{A} \exp \left[-\overleftarrow{\partial}_{\alpha^{*}} \vec{\partial}_{\alpha}\right] P_{B}+\mathcal{O}\left(J^{-1}\right)
\end{aligned}
$$

which recovers the infinite-dimensional case in the large-spin limit if the functions $Q_{A}$, $Q_{B}, P_{A}$, and $P_{B}$ and their differentials remain non-singular in the limit.

(a)

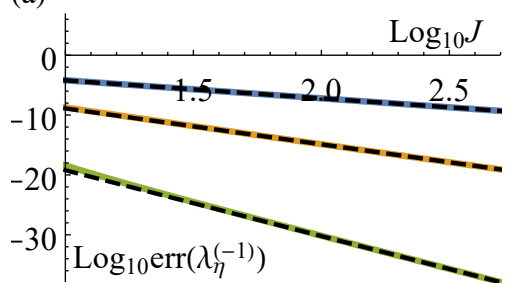

(b)

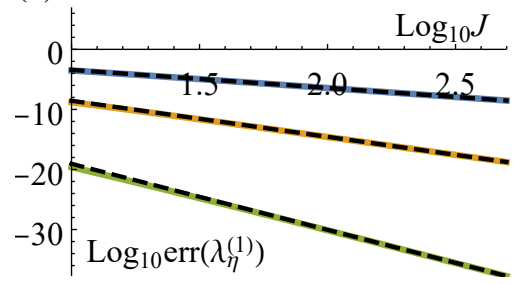

(c)

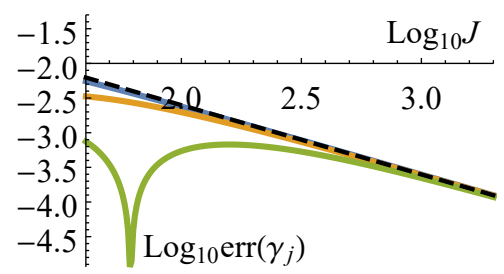

Figure 4. (a)-(b) Difference between the exact factors $\lambda_{\eta}^{(-1)}$ and $\lambda_{\eta}^{(1)}$ from Result 1 (33) and their approximations from (35) and (36) for $\eta \in\{2,5,10\}$ (blue, orange, green). (c) Difference between the exact factor $\gamma_{j}$ and its approximation from Result 4 for $j \in\{2,5,12\}$ (blue, orange, green). Dashed lines show the expected scaling $\mathcal{O}\left(J^{-\eta-1}\right)$ for (a) and (b) as well as $\mathcal{O}\left(J^{-1}\right)$ for (c) with suitable prefactors. 


\section{Transforming between phase-space representations}

\subsection{Exact transformations}

As detailed in [28], the convolution of the finite-dimensional distribution function $F_{\rho}\left(\Omega, s^{\prime}\right)$ with the spin-up state representation $F_{|J J\rangle}(\Omega, s)$ transforms between different representations and results in a type- $\left(s+s^{\prime}-1\right)$ distribution function (refer also to (4))

$$
F_{\rho}\left(\Omega, s+s^{\prime}-1\right)=F_{|J J\rangle}(\theta, s) * F_{\rho}\left(\Omega, s^{\prime}\right)=: \square(s) F_{\rho}\left(\Omega, s^{\prime}\right) .
$$

We rely on (41) to define the differential operator $\square(s)$ which will be often used in the following as a convenient notational shortcut for the convolution. This differential operator satisfies the eigenvalue equation $\square(s) \mathrm{Y}_{j m}=\gamma_{j}^{1-s} \mathrm{Y}_{j m}$ when applied to spherical harmonics 28]. Appendix D details how these eigenvalues $\gamma_{j}^{1-s}$ can be written as a $2 J$ order polynomial in $j(j+1)$ which enables us to specify $\square(s)$ as a polynomial in the differentials ððळ using (19):

Result 3. The operator $\square(s)$ from (41) can be specified as a $2 J$-order polynomial

$$
\square(s) F_{\rho}\left(\Omega, s^{\prime}\right)=\sum_{n=0}^{2 J} c_{n}(s)(ð \bar{\gamma})^{n} F_{\rho}\left(\Omega, s^{\prime}\right)
$$

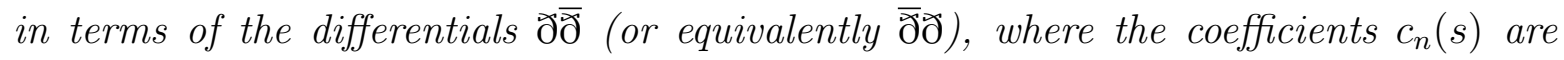
uniquely determined and can be computed analytically (refer to Appendix D for details).

The upper summation bound in 42 can be enlarged to $4 J$ if one performs a truncation of the higher-order spherical-harmonics terms in the resulting phase-space distribution function, refer to Appendix D.

\subsection{Approximate transformations}

The eigenvalue equation for the transformation operator $\square(s)$ from section 7.1 is expanded into

$$
\square(s) \mathrm{Y}_{j m}=\sum_{n=0}^{\infty}\left[-\frac{1-s}{4 J} j(j+1)\right]^{n} / n ! \mathrm{Y}_{j m}+\mathcal{O}\left(J^{-1}\right)
$$

by applying results of Appendix C. This enables the following approximations which are also discussed in figure $4(\mathrm{c})$ :

Result 4. Using the asymptotic expansion from (43), $\square(s)$ can be approximated by

$$
\square(s) F_{\rho}\left(\Omega, s^{\prime}\right)=\exp \left[\frac{1-s}{4 J} \text { ð̄ }\right] F_{\rho}\left(\Omega, s^{\prime}\right)+\mathcal{O}\left(J^{-1}\right) .
$$

Proposition 1 facilitates the approximation

$$
\square(s) F_{\rho}\left(\Omega, s^{\prime}\right)=\exp \left[\frac{1-s}{2} \partial_{\alpha^{*}} \partial_{\alpha}\right] F_{\rho}\left(\Omega, s^{\prime}\right)+\mathcal{O}\left(J^{-1}\right)
$$

in terms of the derivatives $\partial_{\alpha^{*}}$ and $\partial_{\alpha}$. This recovers the infinite-dimensional case from (4) in the large-spin limit if $F_{\rho}\left(\Omega, s^{\prime}\right)$ and its differentials remain non-singular. 


\section{Star products of $s$-parametrized phase spaces}

\subsection{The exact star product}

Generalizing from $\mathrm{P}$ and $\mathrm{Q}$ functions in section 6, the star product of general $s$ parametrized phase spaces is now determined. The differential operator $\square(s)$ can be used to translate the star product of $\mathrm{P}$ or $\mathrm{Q}$ functions to the star product of arbitrary $s$-parametrized distribution functions. In particular, we apply Result 1 to $Q_{A B}=Q_{A} \star^{(-1)} Q_{B}$ and $P_{A B}=P_{A} \star^{(1)} P_{B}$ and use the substitutions

$$
\begin{aligned}
& \square(s+2) Q_{A B}=F_{A B}(\Omega, s), Q_{A}=\square(-s) F_{A}(\Omega, s), Q_{B}=\square(-s) F_{B}(\Omega, s), \\
& \square(s) P_{A B}=F_{A B}(\Omega, s), P_{A}=\square(2-s) F_{A}(\Omega, s), P_{B}=\square(2-s) F_{B}(\Omega, s)
\end{aligned}
$$

from Result 2 in order to compute the star product:

Result 5. The star product of two s-parametrized phase-space distribution functions $F_{A}(\Omega, s)$ and $F_{B}(\Omega, s)$ is given by either of the two equations

$$
\begin{aligned}
& F_{A}(\Omega, s) \star^{(s)} F_{B}(\Omega, s)=\square(s+2)\left\{F_{A}(\Omega, s)\left[\overleftarrow{\square}(-s) \star^{(-1)} \vec{\square}(-s)\right] F_{B}(\Omega, s)\right\} \\
& F_{A}(\Omega, s) \star^{(s)} F_{B}(\Omega, s)=\square(s)\left\{F_{A}(\Omega, s)\left[\overleftarrow{\square}(2-s) \star^{(1)} \vec{\square}(2-s)\right] F_{B}(\Omega, s)\right\} .
\end{aligned}
$$

An explicit expansion can be calculated by expanding $\square(s)$ using (42) and $\star^{( \pm 1)}$ using (32) and by applying the Leibniz identity $\precsim(f g)=(ð f) g+f(ð g)$. This results in an alternative form of the exact star product in (46) and (47):

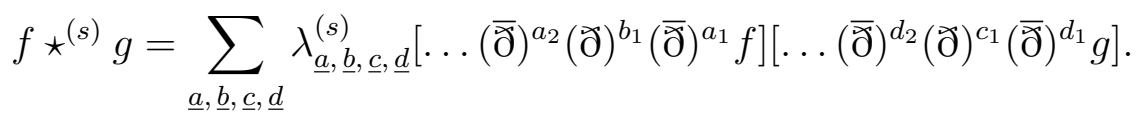

The suitably chosen coefficients $\lambda_{\underline{a}, \underline{b}, \underline{c}, \underline{d}}^{(s)}$ are nonzero only if all of the indices $a_{i}, b_{i}, c_{i}, d_{i}$ are smaller than $2 J+1$. Different values for these coefficients are possible as the product of spin-weight raising and lowering operators can be reordered using their commutators from section 4. But all possible values of the coefficients lead to the same unique result.

Although the choice of the coefficients in the finite sum in 48 is in general not unique due to the non-commutativity of $\widetilde{\partial}$ and $\bar{\partial}$, convenient formulas can be obtained for explicit values of $J$ by reordering products of $ळ$ and $\bar{\varnothing}$. The particular case of $J=1 / 2$ is discussed in section 8.2. For large $J$, the star product can be approximated using the commutative derivatives $\partial_{\alpha^{*}}$ and $\partial_{\alpha}$ from the infinite-dimensional case as discussed in section 8.3. Also, 46 can always be used to calculate the exact star product, but this approach consists of three consecutive steps, as demonstrated in section 9 .

\subsection{The case of a single spin $1 / 2$}

In the particular case of $J=1 / 2$, the exact star product in 46 can be simplified into a more convenient form by applying (48). Let $A$ and $B$ denote spin-1/2 operators and their phase-space representations are given by $f=F_{A}(\Omega, s)$ and $g=F_{B}(\Omega, s)$. The star product is then determined by

$$
f \star^{(s)} g=N_{s} \mathcal{P}\left(f\left[1+a_{s}(\overleftarrow{\bar{\partial}})(\vec{\partial})-b_{s}(\overleftarrow{\check{\partial}})(\overrightarrow{\bar{\partial}})\right] g\right)
$$


where the $s$-dependent coefficients are $N_{s}=2^{-\frac{s}{2}-\frac{1}{2}}$,

$$
a_{s}=\frac{1}{4} 3^{-s-\frac{1}{2}}\left[23^{s / 2}-3^{s+\frac{1}{2}}+\sqrt{3}\right], \text { and } b_{s}=\frac{1}{4} 3^{-s-\frac{1}{2}}\left[23^{s / 2}+3^{s+\frac{1}{2}}-\sqrt{3}\right] .
$$

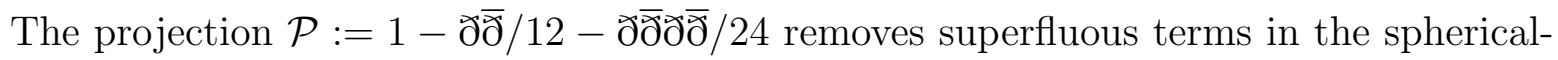
harmonics decomposition, i.e., contributions $\mathrm{Y}_{j m}$ with $j>1$ that do not correspond to spin-1/2 distribution functions (refer to Result 2 in [34]). Note that for Wigner functions (i.e. $s=0$ ) the explicit form of the star product can be calculated as (see, e.g., 34)

$$
W_{A} \star^{(0)} W_{B}=\mathcal{P} R\left[\sqrt{2 \pi} W_{A} W_{B}-\frac{i}{2} \sqrt{\frac{8 \pi}{3}}\left\{W_{A}, W_{B}\right\}_{S}\right]
$$

where $a_{0}=b_{0}=1 /(2 \sqrt{3})$ and $N_{0}=1 / \sqrt{2}$. For $J=1 / 2$, we have the radius $R=(4 \pi)^{-1 / 2}$ and the spherical Poisson bracket has the form $i\{., .\}_{S}=\overleftarrow{\partial}_{\phi}(\sin \theta)^{-1} \vec{\partial}_{\theta}-$ $\overleftarrow{\partial}_{\theta}(\sin \theta)^{-1} \vec{\partial}_{\phi}$, which should also be compared to 53 .

\subsection{Approximations of the star product}

Applying Result 2 and Result 4, the exact star product in Result 5 can be efficiently approximated as detailed in the following:

Result 6. Let $f=F_{A}(\Omega, s)$ and $g=F_{B}(\Omega, s)$ denote the phase-space functions of the spin-J operators $A$ and $B$. The star product in (46) can be expanded in terms of spin-weight raising and lowering operators as (refer to Appendix $F$ for a proof)

$$
f \star^{(s)} g=\sum_{n=0}^{4 J} \sum_{m=0}^{n} \frac{c_{n m}(s)}{(2 J)^{n}}\left[\bar{ð}^{m} \check{\partial}^{n-m} f\right]\left[\check{\partial}^{m} \overline{\check{\partial}}^{n-m} g\right]+\mathcal{O}\left(J^{-1}\right),
$$

where the coefficients $c_{n m}(s)$ are defined in Appendix F. Similarly, the star product can be specified in terms of the derivatives

$$
f \star^{(s)} g=f \exp \left[\frac{(1-s)}{2} \overleftarrow{\partial}_{\alpha} \vec{\partial}_{\alpha^{*}}-\frac{(1+s)}{2} \overleftarrow{\partial}_{\alpha^{*}} \vec{\partial}_{\alpha}\right] g+\mathcal{O}\left(J^{-1}\right)
$$

The infinite-dimensional case from Equation (7) is recovered in the large-spin limit by applying Proposition 1 if $f$ and $g$ and their differentials remain non-singular.

Note that the first-order term (i.e. $n=1$ ) of the star product $\star^{(0)}$ in 51 is for the case of a Wigner function (i.e. $s=0$ ) proportional to the spherical Poisson bracket [34]

$$
\{., .\}_{S}:=i[(\overleftarrow{\bar{\partial}})(\vec{\partial})-(\overleftarrow{\check{\partial}})(\overrightarrow{\bar{\partial}})] /(2 J)=\overleftarrow{\partial}_{\phi}(2 J \sin \theta)^{-1} \vec{\partial}_{\theta}-\overleftarrow{\partial}_{\theta}(2 J \sin \theta)^{-1} \vec{\partial}_{\phi}
$$

which corresponds to the classical part of the time evolution [34]. The approximate star product can be also used to derive efficient approximations of finite-dimensional phase-space representations for large $J$ as illustrated in section 10 .

\section{Time evolution of quantum states for a single spin $J$}

\subsection{Description of the time evolution using the star product}

The time evolution of a quantum state $\rho$ for a single spin $J$ can be described in a phase space via the Moyal equation from (2). We discuss now the general structure of the 
time evolution of phase-space functions along the lines of [34] and present an explicit example in section 9.2 . We refer to 34 for further background and additional examples. Substituting the $s$-parametrized star product in (2) with one of its forms from Result 5 yields the exact equation of motion for an arbitrary quantum state $F_{\rho}(\Omega, s)$ under a Hamiltonian $F_{\mathcal{H}}(\Omega, s)$ as (refer, e.g., (47))

$$
\begin{aligned}
\frac{\partial F_{\rho}(\Omega, s)}{\partial t}= & -i \square(s)\left\{F_{\mathcal{H}}(\Omega, s)\left[\overleftarrow{\square}(2-s) \star^{(1)} \vec{\square}(2-s)\right] F_{\rho}(\Omega, s)\right\} \\
& +i \square(s)\left\{F_{\rho}(\Omega, s)\left[\overleftarrow{\square}(2-s) \star^{(1)} \vec{\square}(2-s)\right] F_{\mathcal{H}}(\Omega, s)\right\}
\end{aligned}
$$

The use of this equation is illustrated in section 9.2 with the particular case of a Wigner function $(s=0)$. One can approximate this time evolution by substituting the $s$ parametrized star product in (2) with one of its approximations from Result 6. This yields for $f:=F_{\mathcal{H}}(\Omega, s)$ and $g:=F_{\rho}(\Omega, s)$ an approximate equation of motion up to an error of order $\mathcal{O}\left(J^{-1}\right)$ as (e.g.)

$$
\frac{\partial F_{\rho}(\Omega, s)}{\partial t} \approx \sum_{n=1}^{4 J} \sum_{m=0}^{n} \frac{c_{n m}(s)}{(2 J)^{n}}\left\{-i\left[\overline{\mathrm{\partial}}^{m} \check{\partial}^{n-m} f\right]\left[\check{\partial}^{m} \overline{\mathrm{\partial}}^{n-m} g\right]+i\left[\overline{\mathrm{\partial}}^{m} \check{\partial}^{n-m} g\right]\left[\check{\partial}^{m} \overline{\mathrm{\delta}}^{n-m} f\right]\right\} .
$$

Note that the first term of the summation $(n=1)$ coincides with the spherical Possion bracket from (53) for the special case of Wigner functions $(s=0)$ and corresponds to a semiclassical time evolution [34]. These semiclassical approximations have been widely used, refer to, e.g., 23, 25, 41]. Higher order contributions $n \geq 2$ are used to approximate quantum contributions in the time evolution.

\subsection{Example of an explicit and exact time evolution for a single spin $J$}

We discuss in this section an example for a single spin with arbitrary spin number $J$ which illustrates the application of the exact star product from (46). Let us consider an experimental system with a single spin $J$ that is controlled as, e.g., in solid state nuclear magnetic resonance [60]. The density operator of a single spin $J$ is in the thermal equilibrium given by $\rho_{0} \propto \mathbb{1}+\beta \mathcal{I}_{z}$ where $\beta$ depends on the temperature. (We use the notations $\mathcal{I}_{z}=\mathrm{T}_{10} /\left(\sqrt{2} N_{J}\right)$ and $\mathcal{I}_{+}=\mathrm{T}_{11} / N_{J}$ with $N_{J}=1 / \sqrt{2 J(J+1)(2 J+1) / 3}$. $) \mathrm{We}$ assume an effective Hamiltonian of the form $\mathcal{H}_{\text {eff }}:=\omega\left(\mathcal{I}_{+}\right)^{3}+\mathcal{H}_{\text {res }}$. The first-order time evolution under this effective Hamiltonian is given by the von-Neumann equation $\frac{\partial \rho_{0}}{\partial t}=-i \omega\left[\left(\mathcal{I}_{+}\right)^{3}, \mathcal{I}_{z}\right]-i\left[\mathcal{H}_{\text {res }}, \rho_{0}\right]$, and the commutator $\left[\left(\mathcal{I}_{+}\right)^{3}, \mathcal{I}_{z}\right]$ is proportional to $\left(\mathcal{I}_{+}\right)^{3}$. The term $\left(\mathcal{I}_{+}\right)^{3}$ is responsible for creating multiple quantum coherences which are often desirable. One can design experimental controls that maximize this contribution in the effective Hamiltonian [60].

Equivalently, the time evolution of the density operator $\rho:=T_{10}$ under the Hamiltonian $\mathcal{H}:=\mathrm{T}_{33}$ can be calculated for a single spin with arbitrary spin number $J$ directly in a phase-space representation. The corresponding spin Wigner functions $W_{\mathcal{H}}:=\frac{1}{R} Y_{33}(\theta, \phi)$ and $W_{\rho}:=\frac{1}{R} Y_{10}(\theta, \phi)$ are specified in terms of spherical harmonics, see figure 5 (a). The time evolution of the Wigner function $W_{\rho}$ is described in the phase 
(b)

$$
\frac{\partial W_{\rho}}{\partial t}=-i\left(W_{\mathcal{H}} \star^{(0)} W_{\rho}-W_{\rho} \star^{(0)} W_{\mathcal{H}}\right)=\frac{-i x_{2}}{\gamma_{3} \gamma_{1} R^{2}}
$$

(a)

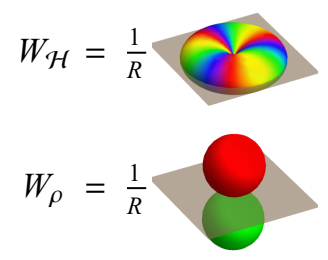

(d) (h)

(c)

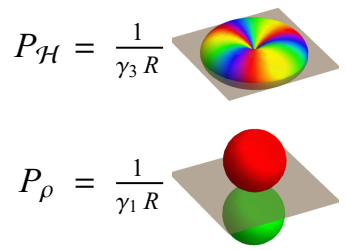

(i)

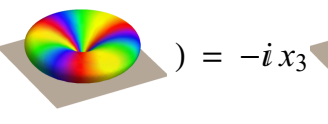

(g)

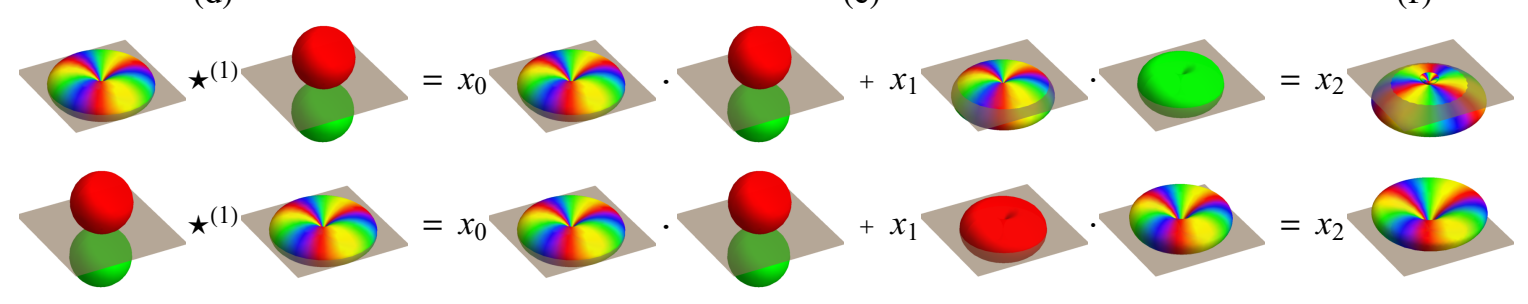

Figure 5. Visualizing the calculation of the exact time evolution using the star product of Wigner functions for a single spin with an arbitrary spin number J: (a) $W_{\mathcal{H}}:=\frac{1}{R} \mathrm{Y}_{33}(\theta, \phi)$ and $W_{\rho}:=\frac{1}{R} \mathrm{Y}_{10}(\theta, \phi)$ and (c) their corresponding $\mathrm{P}$ functions, (b) time evolution in the phase space via the Moyal equation $\sqrt{56}$, (d)-(f) star products $P_{\mathcal{H}} \star^{(1)} P_{\rho}$ and $P_{\rho} \star^{(1)} P_{\mathcal{H}}$ using $(58)-(59),(\mathrm{g})$ the resulting star products decompose into two spherical harmonics as in 60); (h) substituting (f) back into the Moyal equation (b) leads to (i) where the symmetric contribution from $(\mathrm{g})$ is canceled out. The prefactors $x_{0}, x_{1}, x_{2}, x_{3}$ are used to rescale some of the plots.

space by the Moyal equation (cf. [34])

$$
\frac{\partial W_{\rho}}{\partial t}=-i W_{\mathcal{H}} \star^{(0)} W_{\rho}+i W_{\rho} \star^{(0)} W_{\mathcal{H}}
$$

by relying on the star commutator which is determined in terms of star products, see figure 5(b). The star product $W_{\mathcal{H}} \star^{(0)} W_{\rho}$ is evaluated via Result 5 . Using (47), we get

$$
W_{\mathcal{H}} \star^{(0)} W_{\rho}=\square(0)\left(W_{\mathcal{H}}\left[\overleftarrow{\square}(2) \star^{(1)} \vec{\square}(2)\right] W_{\rho}\right)
$$

where $\square(2)$ is determined by Result 3 (refer to Equation (41)) and transforms the Wigner functions to the corresponding $\mathrm{P}$ functions $P_{\mathcal{H}}=W_{\mathcal{H}} \stackrel{\leftarrow}{\square}(2)=\frac{1}{R \gamma_{3}} \mathrm{Y}_{33}(\theta, \phi)$ and $P_{\rho}=\vec{\square}(2) W_{\rho}=\frac{1}{R \gamma_{1}} \mathrm{Y}_{10}(\theta, \phi)$, see figure 5(c). Note that spherical harmonics $\mathrm{Y}_{j m}$ are eigenfunctions of $\square(2)$ with eigenvalues $\gamma_{j}^{-1}$. The right hand side of (57) is then equal to $\square(0)\left(P_{\mathcal{H}} \star^{(1)} P_{\rho}\right)$, for which the star product of $\mathrm{P}$ functions is computed using 32 in Result 1. This yields (see figure $5(\mathrm{~d}-\mathrm{f})$ )

$$
\begin{aligned}
& P_{\mathcal{H}} \star^{(1)} P_{\rho}=\frac{\mathrm{Y}_{33 \star^{(1)}} \mathrm{Y}_{10}}{R^{2} \gamma_{1} \gamma_{3}}=\frac{1}{R^{2} \gamma_{1} \gamma_{3}}\left[\lambda_{0}^{(1)} \mathrm{Y}_{33} \mathrm{Y}_{10}+\lambda_{1}^{(1)}\left(ð \mathrm{Y}_{33}\right)\left(\overline{\widetilde{\partial}} \mathrm{Y}_{10}\right)\right], \\
& P_{\mathcal{H}} \star^{(1)} P_{\rho}=\frac{1}{R^{2} \gamma_{1} \gamma_{3}}\left[\lambda_{0}^{(1)} \mathrm{Y}_{33} \mathrm{Y}_{10}-\lambda_{1}^{(1)} 2 \sqrt{6} \mathrm{Y}_{33}^{1} \mathrm{Y}_{10}^{-1}\right] .
\end{aligned}
$$

The coefficients $\lambda_{0}^{(1)}$ and $\lambda_{1}^{(1)}$ are defined in $(33)$ and the operators $\partial$ and $\bar{\partial}$ from 16 are responsible for raising and lowering the spin weight of the spherical harmonics. 
Products of spin-weighted spherical harmonics can be decomposed into sums $\mathrm{Y}_{33} \mathrm{Y}_{10}=\frac{1}{2 \sqrt{3 \pi}} \mathrm{Y}_{43}$ and $\mathrm{Y}_{33}^{1} \mathrm{Y}_{10}^{-1}=-\frac{3}{4 \sqrt{2 \pi}} \mathrm{Y}_{33}+\frac{1}{4 \sqrt{2 \pi}} \mathrm{Y}_{43}$ of spherical harmonics. The corresponding star product of Wigner functions is obtained by rescaling spherical harmonics $\mathrm{Y}_{j m}$ by $\gamma_{j}$, which results in (refer to figure $5(\mathrm{~g}-\mathrm{h})$ )

$$
\begin{aligned}
& W_{\mathcal{H}} \star^{(0)} W_{\rho}=\frac{1}{R^{2} \gamma_{1} \gamma_{3}}\left[+\frac{\lambda_{1}^{(1)} 3 \sqrt{3}}{2 \sqrt{\pi}} \gamma_{3} \mathrm{Y}_{33}+\left(\frac{\lambda_{0}^{(1)}}{2 \sqrt{3 \pi}}-\frac{\lambda_{1}^{(1)} \sqrt{3}}{2 \sqrt{\pi}}\right) \gamma_{4} \mathrm{Y}_{43}\right] \\
& W_{\rho} \star^{(0)} W_{\mathcal{H}}=\frac{1}{R^{2} \gamma_{1} \gamma_{3}}\left[-\frac{\lambda_{1}^{(1)} 3 \sqrt{3}}{2 \sqrt{\pi}} \gamma_{3} \mathrm{Y}_{33}+\left(\frac{\lambda_{0}^{(1)}}{2 \sqrt{3 \pi}}-\frac{\lambda_{1}^{(1)} \sqrt{3}}{2 \sqrt{\pi}}\right) \gamma_{4} \mathrm{Y}_{43}\right] .
\end{aligned}
$$

Note that $\gamma_{4}=0$ for $J<2$ which is responsible for truncating the spherical-harmonics decomposition [34, refer to Appendix D. The final result determining the time evolution is obtained via the star commutator from (56), and we obtain (for arbitrary $\mathrm{J}$ )

$$
\frac{\partial W_{\rho}}{\partial t}=-i \frac{3 \sqrt{3}}{\sqrt{\pi}} \frac{\lambda_{1}^{(1)}}{R^{2} \gamma_{1}} \mathrm{Y}_{33}
$$

\subsection{Extending the example to an arbitrary quantum state}

Applying the same Hamiltonian $W_{\mathcal{H}}=\frac{1}{R} Y_{33}$ to an arbitrary quantum state $g:=W_{\rho}$, we could apply (54) to determine the time evolution exactly. But we will consider here only the approximate time evolution (see (55))

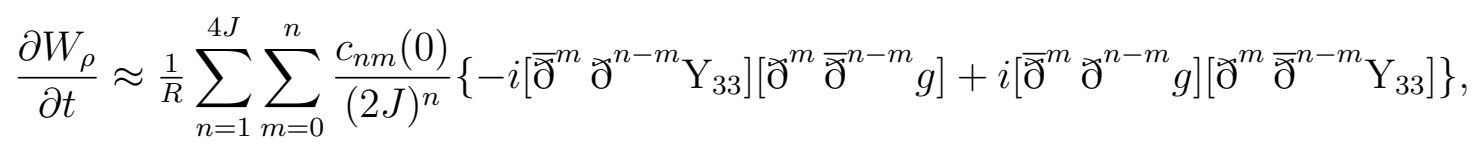

where one can apply (15) to simplify differentials. For example, one obtains (up to a factor) the spin-weighted spherical harmonics

$$
\bar{\delta}^{m} \partial^{n-m} Y_{33} \propto \begin{cases}Y_{33}^{n-2 m} & \text { for }|n-m| \leq 3 \text { and }|n-2 m| \leq 3 \\ 0 & \text { otherwise. }\end{cases}
$$

This highlights that most of the terms in the sum vanish for a general spin number $J$. But this example makes it also apparent that a semiclassical approximation [23, 25, 41] that restricts the summation to $n=1$ will neglect relevant quantum contributions.

\section{One example of photon-added coherent states}

Creation and annihilation operators are widely used and account for numerous nonclassical effects including, for example, photon-added coherent states which were demonstrated experimentally [61 66]. Photon-added coherent states are obtained from coherent states $\left|\alpha_{0}\right\rangle:=\mathcal{D}\left(\alpha_{0}\right)|0\rangle$ as $a^{\dagger}\left|\alpha_{0}\right\rangle$ by applying the creation operator $a^{\dagger}$. The inversely translated version of these quantum states is created from the vacuum state by applying the operator $Q\left(\alpha_{0}\right)$ and one has

$$
\left|\alpha_{0}^{+}\right\rangle=Q\left(\alpha_{0}\right)|0\rangle \text { with } Q\left(\alpha_{0}\right):=\frac{1}{\sqrt{1+\left|\alpha_{0}\right|^{2}}} \mathcal{D}\left(\alpha_{0}\right)^{-1} a^{\dagger} \mathcal{D}\left(\alpha_{0}\right) .
$$

Phase-space representations of these photon-added coherent states can be obtained using the star products of the individual phase-space representations

$$
F_{\left|\alpha_{0}^{+}\right\rangle}(\alpha, s)=F_{Q\left(\alpha_{0}\right)} \star^{(s)} F_{|0\rangle}(\alpha, s) \star^{(s)}\left(F_{Q\left(\alpha_{0}\right)}\right)^{*},
$$




$$
J=\frac{3}{2} \quad J=4 \quad J=10 \quad J \rightarrow \infty
$$

(d)

(a)
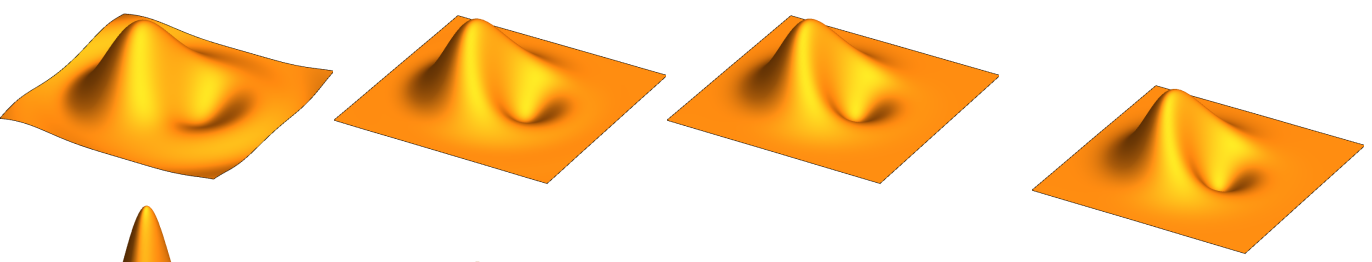

(b)

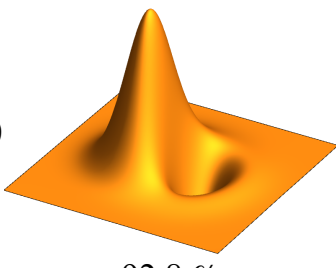

$92.8 \%$
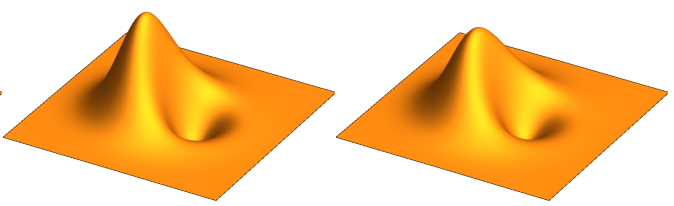

(e)

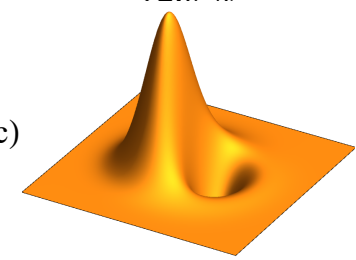

$85.0 \%$

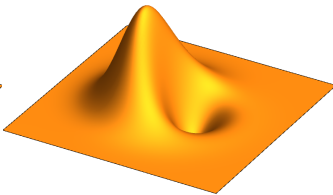

$29.0 \%$
$11.6 \%$

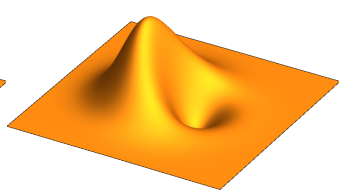

$11.2 \%$

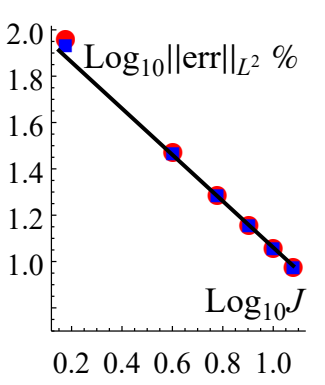

Figure 6. (a) Plots of the exact phase-space representations $F_{\left|\Omega_{0}^{+}\right\rangle}(\Omega, 0)$ of $\left|\Omega_{0}^{+}\right\rangle$ for increasing spin numbers $J$ using $\sqrt[69]{60}$. (b)-(c) Approximations of $F_{\left|\Omega_{0}^{+}\right\rangle}(\Omega, 0)$ using (71) and the Gaussian function $F_{|J J\rangle}(\Omega, 0)$, also applying the approximate star product in (51) for (b) and (52) for (c). Both (b) and (c) converge to (a) in order $\mathcal{O}\left(J^{-1}\right)$ as they approach their infinite-dimensional counterpart (d) from (64). The approximation errors in the $L^{2}$ norm shown below the plots are graphed in (e) for $J \in\{3 / 2,4,6,8,10,12\}$, where red dots and blue squares refer, respectively, to (b) and (c) and the black line shows the expected scaling $\mathcal{O}\left(J^{-1}\right)$ with a suitable prefactor. The distance from the origin in these planar plots represent the arc distance from the north pole in the spherical phase space. The rotation parameter in $(69)$ has been chosen as $\Omega_{0}=\left(\theta_{0}, \phi_{0}\right)=(1.2 / \sqrt{J / 2}, 0)$ and the translation parameter in (64) is given by $\alpha_{0}=1.2$. Plot limits are \pm 2 . Refer also to Figure 1 and Figure 7 .

where the Gaussian function $F_{|0\rangle}(\alpha, s)=2 \exp \left[-2 \alpha \alpha^{*} /(1-s)\right] /(1-s)$ represents the vacuum state $\left[15\right.$ and $F_{Q\left(\alpha_{0}\right)}:=\left(\alpha^{*}+\alpha_{0}^{*}\right) / \sqrt{1+\left|\alpha_{0}\right|^{2}}$ corresponds to the creation operator. Applying the star product from (7) yields

$$
F_{\left|\alpha_{0}^{+}\right\rangle}(\alpha, s)=\left[\alpha+\alpha_{0}-\frac{1+s}{2} \partial_{\alpha^{*}}\right]\left[\alpha^{*}+\alpha_{0}^{*}-\frac{1+s}{2} \partial_{\alpha}\right] F_{|0\rangle}(\alpha, s)=: \overline{\mathcal{Q}}\left(\alpha_{0}\right) \mathcal{Q}\left(\alpha_{0}\right) F_{|0\rangle}(\alpha, s),
$$

where the second equality describes the photon creation in the shifted phase space in terms of the differential operators $\mathcal{Q}\left(\alpha_{0}\right) \overline{\mathcal{Q}}\left(\alpha_{0}\right)=\overline{\mathcal{Q}}\left(\alpha_{0}\right) \mathcal{Q}\left(\alpha_{0}\right)$. Setting $\alpha_{0}=0$ yields the phase-space equivalent of the creation operator, i.e., essentially Bopp operators 67]

$$
\mathcal{Q}:=\mathcal{Q}(0)=\left[\alpha^{*}-\frac{1+s}{2} \partial_{\alpha}\right] \text { and } \overline{\mathcal{Q}}:=\overline{\mathcal{Q}}(0)=\left[\alpha-\frac{1+s}{2} \partial_{\alpha^{*}}\right]
$$

For the number state $|n\rangle$, one can calculate the phase-space functions

$$
F_{|n\rangle}(\alpha, s)=\frac{1}{n !}[\overline{\mathcal{Q}} \mathcal{Q}]^{n} F_{|0\rangle}(\alpha, s) \text { and } F_{\left|n_{1}\right\rangle\left\langle n_{2}\right|}(\alpha, s)=\frac{1}{\sqrt{n_{1} !} \sqrt{n_{2} !}}(\overline{\mathcal{Q}})^{n_{2}}(\mathcal{Q})^{n_{1}} F_{|0\rangle}(\alpha, s)
$$

corresponds to tilted projectors $\left|n_{1}\right\rangle\left\langle n_{2}\right|$ which span a complete, orthonormal basis [15]. 
(a)
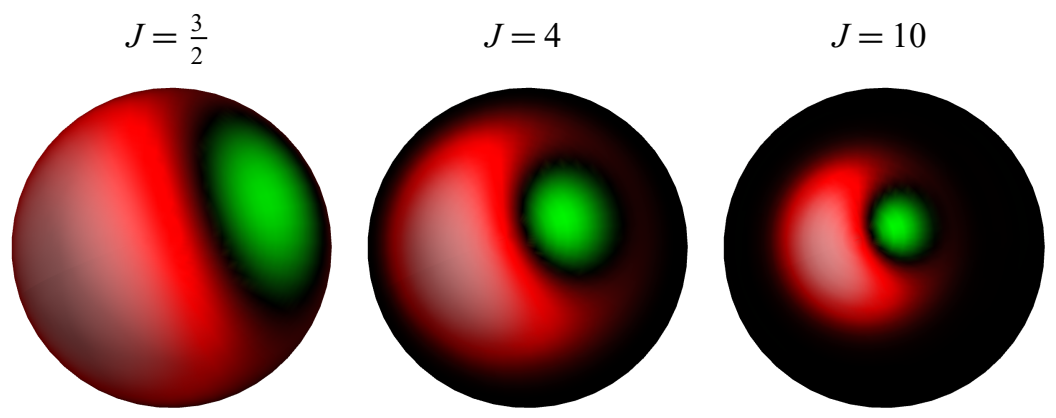

(b)
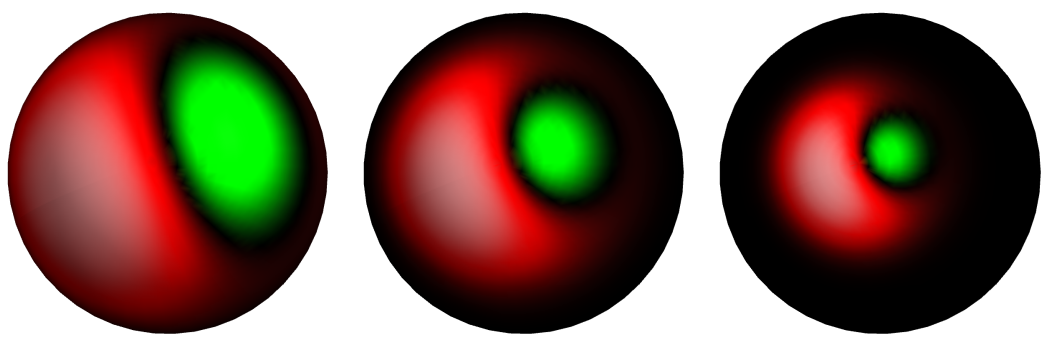

Figure 7. Alternative representation of figure 6(a)-(b). (a) Plots of the exact phase-space representations $F_{\left|\Omega_{0}^{+}\right\rangle}(\Omega, 0)$ of $\left|\Omega_{0}^{+}\right\rangle$for increasing $J$ using $\sqrt[69]{ }$. (b) Approximations of $F_{\left|\Omega_{0}^{+}\right\rangle}(\Omega, 0)$ via $\sqrt{71}$ and 51 assuming a Gaussian $F_{|J J\rangle}(\Omega, 0)$. (b) converges to (a) in order $\mathcal{O}\left(J^{-1}\right)$. Spherical functions $f(\theta, \phi)$ are plotted on the sphere where the brightness represents the absolute value and the colors red (dark gray) and green (light gray) depict positive and negative values, respectively.

We define finite-dimensional analogues of photon-added coherent states in the form

$$
\left|\Omega_{0}^{+}\right\rangle:=K\left(\Omega_{0}\right)|J J\rangle \text { with } K\left(\Omega_{0}\right):=\frac{N}{\sqrt{2 J}} \mathcal{R}^{-1}\left(\Omega_{0}\right) \mathcal{J}_{-} \mathcal{R}\left(\Omega_{0}\right),
$$

where $|J J\rangle$ is the spin-up state and $\mathcal{J}_{-} / \sqrt{2 J}$ is the finite-dimensional analogue of the creation operator which approaches $a^{\dagger}$ in the large-spin limit. Refer to Table 1 in [49] and Appendix G for details. Following the infinite-dimensional characterization, the phase-space representation $F_{\left|\Omega_{0}^{+}\right\rangle}$can be written in terms of the exact star product of the individual phase-space representations as (see Result 5)

$$
F_{\left|\Omega_{0}^{+}\right\rangle}(\Omega, s)=F_{K\left(\Omega_{0}\right)} \star^{(s)} F_{|J J\rangle}(\Omega, s) \star^{(s)}\left(F_{K\left(\Omega_{0}\right)}\right)^{*},
$$

where the Gaussian-like function $F_{|J J\rangle}(\Omega, s)$ represents the spin-up state and $F_{K\left(\Omega_{0}\right)}$ is the phase-space representation of the rotated lowering operator $K\left(\Omega_{0}\right)$. Refer to Figure 6(a) for plots of the (unapproximated) $F_{\left|\Omega_{0}^{+}\right\rangle}(\Omega, s)$ for $s=0$. Using, e.g., the exact star product in (46), the phase-space representation

$$
F_{\left|\Omega_{0}^{+}\right\rangle}(\Omega, s)=\mathcal{K}\left(\Omega_{0}\right) \overline{\mathcal{K}}\left(\Omega_{0}\right) F_{|J J\rangle}(\Omega, s)=\overline{\mathcal{K}}\left(\Omega_{0}\right) \mathcal{K}\left(\Omega_{0}\right) F_{|J J\rangle}(\Omega, s)
$$

of the excited coherent state $F_{\left|\Omega_{0}^{+}\right\rangle}(\Omega, s)$ is obtained by applying the differential operators $\mathcal{K}\left(\Omega_{0}\right)$ and $\overline{\mathcal{K}}\left(\Omega_{0}\right)$ which are defined via their action on phase space functions $f$, i.e.,

$$
\mathcal{K}\left(\Omega_{0}\right) f:=F_{K\left(\Omega_{0}\right)} \star^{(s)} f \text { and } \overline{\mathcal{K}}\left(\Omega_{0}\right) f:=f \star^{(s)}\left(F_{K\left(\Omega_{0}\right)}\right)^{*} .
$$

Approximations of these operators can be used to approximate $F_{\left|\Omega_{0}^{+}\right\rangle}(\Omega, s)$ by applying them to a Gaussian approximation of $F_{|J J\rangle}(\Omega, s)$ as given in 28$]$. Approximations of 
$\mathcal{K}\left(\Omega_{0}\right)$ and $\overline{\mathcal{K}}\left(\Omega_{0}\right)$ are calculated in terms of spin-weighted spherical harmonics and their raising and lowering operators in Appendix G using the approximate star products in (51) and (52). These approximations converge in figure 6 to the exact phase-space functions, and the infinite-dimensional case from (64) is recovered in the large-spin limit.

Comparable to the infinite-dimensional case, the unrotated ladder operator $\mathcal{K}:=$ $\mathcal{K}(0)$ is specified in Appendix $\mathrm{G}$ in terms of spin-weighted spherical harmonics and their raising and lowering operators. One can calculate representations

$$
F_{|J m\rangle}(\Omega, s) \propto[\mathcal{K} \overline{\mathcal{K}}]^{J-m} F_{|J J\rangle}(\Omega, s)
$$

of the Dicke state $|J m\rangle$ using the operators $\mathcal{K}$ and $\overline{\mathcal{K}}$, and similar representations

$$
F_{\left|J m_{1}\right\rangle\left\langle J m_{2}\right|}(\Omega, s) \propto(\overline{\mathcal{K}})^{J-m_{2}}(\mathcal{K})^{J-m_{1}} F_{|J J\rangle}(\Omega, s)
$$

are obtained for the tilted projectors $\left|J m_{1}\right\rangle\left\langle J m_{2}\right|$. All of these states have typically quite complicated spherical-harmonics expansions which are challenging to calculate for large values of $J$. Approximations based on the star product in (52) facilitate efficient calculations of these and similar phase-space representations for large $J$.

We want to close this section by remarking that general (infinite-dimensional) $s$ parametrized phase spaces naturally appear in experimental homodyne measurements [3, 61 65] of the discussed photon-added coherent states. As is explained in 68], the relevant experiment yields $s$-parametrized phase-space functions with $s=-(1-\xi) / \xi$ for a detector efficiency of $\xi$. This provides an example for the occurrence of $s$-parametrized phase spaces beyond the particular cases of $s \in\{-1,0,1\}$.

\section{Generalization to coupled spins}

The explicit form of the star product for Wigner functions of coupled spin- $1 / 2$ systems was detailed in Result 3 of [34]. Building on results in [34], we outline how to generalize these results to $s$-parameterized phase spaces of coupled spins $J$. We consider two operators $A$ and $B$ in a system of $N$ coupled spins $J$. Their phase-space representations are determined similarly as in Result 1 of [34] and can be calculated as

$$
F_{A}\left(s, \theta_{1}, \phi_{1}, \ldots, \theta_{N}, \phi_{N}\right):=\operatorname{tr}\left[A \bigotimes_{k=1}^{N} \mathcal{R}\left(\theta_{k}, \phi_{k}\right) M_{s} \mathcal{R}^{\dagger}\left(\theta_{k}, \phi_{k}\right)\right],
$$

where the transformation kernel in Result 1 of [34] is expressed here in terms of rotated parity operators from (8). We generalize the star product described in Result 5 to the star product

$$
F_{A} \star F_{B}:=F_{A}\left(\prod_{k=1}^{N}\left[\star^{(s)}\right]^{\{k\}}\right) F_{B}
$$

of phase-space representations for coupled spins by applying Result 3 of [34], where $\star^{(s)}$ is the star product from Result 5 and $\left[\star^{(s)}\right]^{\{k\}}$ describes that the star product acts only on the variables $\theta_{k}$ and $\phi_{k}$. Equation (76) completely specifies the exact star product for a system of $N$ interacting spins $J$, and the corresponding approximations via 
Result 6 can be conveniently expressed using the commutativity of partial derivatives. For example, the approximate star product in terms of the derivatives $\partial_{\alpha}$ and $\partial_{\alpha^{*}}$ from (52) is generalized for coupled spins to

$$
F_{A} \star^{(s)} F_{B}=F_{A} \exp \left[\sum_{k=1}^{N}\left(\frac{(1-s)}{2} \overleftarrow{\partial}_{\alpha_{k}} \vec{\partial}_{\alpha_{k}^{*}}-\frac{(1+s)}{2} \overleftarrow{\partial}_{\alpha_{k}^{*}} \vec{\partial}_{\alpha_{k}}\right)\right] F_{B}+\mathcal{O}\left(J^{-1}\right)
$$

The equation of motion for Wigner functions, i.e., the Moyal equation from (56), can be consequently established for a system of coupled spins $J$ using (77) as

$$
i \frac{\partial W_{\rho}}{\partial t}=W_{\mathcal{H}} \star^{(0)} W_{\rho}-W_{\rho} \star^{(0)} W_{\mathcal{H}}=W_{\mathcal{H}}\left[e^{i\{, \ldots\} / 2}-e^{-i\{,,\} / 2}\right] W_{\rho}+\mathcal{O}\left(J^{-1}\right),
$$

where $\{.,\}:.=\sum_{k=1}^{N} g_{k}$ and $g_{k}:=-i \overleftarrow{\partial}_{\alpha_{k}} \vec{\partial}_{\alpha_{k}^{*}}+i \overleftarrow{\partial}_{\alpha_{k}^{*}} \vec{\partial}_{\alpha_{k}}$ specify a Poisson bracket acting on the variables $\alpha_{k}$ and $\alpha_{k}^{*}$. This results in the expansion

$$
\begin{aligned}
i \frac{\partial W_{\rho}}{\partial t} & =W_{\mathcal{H}}\left[2 \sum_{\substack{n=0, n \text { odd }}}(-i\{., .\} / 2)^{n} / n !\right] W_{\rho}+\mathcal{O}\left(J^{-1}\right) \\
& =W_{\mathcal{H}}\left[-i \sum_{k=1}^{N} g_{k}+\frac{i}{24} \sum_{k_{1}, k_{2}, k_{3}=1}^{N} g_{k_{1}} g_{k_{2}} g_{k_{3}}+\cdots\right] W_{\rho}+\mathcal{O}\left(J^{-1}\right) .
\end{aligned}
$$

Using Proposition 1, the differential operators $-g_{k}$ can be replaced by the spherical Poisson brackets $p_{k}:=\{., .\}_{S}^{\{k\}}$ from $(53)$, which results in the time evolution

$$
i \frac{\partial W_{\rho}}{\partial t}=W_{\mathcal{H}}\left[i \sum_{k=1}^{N} p_{k}-\frac{i}{24} \sum_{\substack{k_{1}, k_{2}, k_{3}=1 \\ k \neq \neq k_{\nu} \text { for } \mu \neq \nu}}^{N} p_{k_{1}} p_{k_{2}} p_{k_{3}}+\cdots-\frac{i}{24} \sum_{k=1}^{N} p_{k}^{3}+\cdots\right] W_{\rho}+\mathcal{O}\left(J^{-1}\right) .
$$

The first two terms (before the first dots) can be directly compared to the ones appearing in the star product of coupled spins $1 / 2$ in Result 4 of [34]. The leading term corresponds to the classical equation of motion, and the following terms in the expansion are ordered according to their degree of non-locality as proposed in Result 4 of 34 .

\section{Conclusion}

We have derived the exact star product for continuous s-parametrized phase-space representations of single spins $J$ in terms of spin-weighted spherical harmonics and their raising and lowering operators. Our construction naturally recovers the well-known case of infinite-dimensional quantum systems in the limit of large spin numbers $J$. Based on approximations of spin-weighted spherical harmonics, we have derived convenient formulas for approximating star products which, beyond the time evolution, can be useful for efficiently calculating phase-space representations for large spin numbers. We have illustrated our methods and their application in concrete examples. We have finally outlined how the presented formalism can be extended to coupled spin systems. In summary, we have established a complete phase-space description for finite-dimensional quantum systems and their time evolution. 


\section{Acknowledgments}

B.K. acknowledges financial support from the scholarship program of the Bavarian Academic Center for Central, Eastern and Southeastern Europe (BAYHOST). R.Z. and S.J.G. acknowledge support from the Deutsche Forschungsgemeinschaft (DFG) through Grant No. Gl 203/7-2.

\section{Appendix A. Expansions of products of tensor operators}

Adopting the notation of [34], the product of two irreducible tensor operators can besimilarly as in (12) - expanded as (see [69])

$$
\mathrm{T}_{j_{1} m_{1}} \mathrm{~T}_{j_{2} m_{2}}=\sum_{L=\left|j_{1}-j_{2}\right|}^{n}{ }^{J} Q_{j_{1} j_{2} L} C_{j_{1} m_{1} j_{2} m_{2}}^{L M} \mathrm{~T}_{L M}
$$

The upper limit $n:=\min \left(j_{1}+j_{2}, 2 J\right)$ of the summation is bounded by $2 J$. We have set $M:=m_{1}+m_{2}$ and also use the Clebsch-Gordan coefficients $C_{j_{1} m_{1}, j_{2} m_{2}}^{L M}$ [47. The coefficients ${ }^{J} Q_{j_{1} j_{2} L}$ from 34 are proportional to Wigner 6- $j$ symbols [47 and depend only on $j_{1}, j_{2}$, and $L$, but are independent of $m_{1}, m_{2}$, and $M$.

Similarly, the product of any two spin-weighted spherical harmonics can be decomposed into a sum of spin-weighted spherical harmonics (see Eq. 2.54 in [59])

$$
\begin{aligned}
\mathrm{Y}_{j_{1} m_{1}}^{\eta_{1}} \mathrm{Y}_{j_{2} m_{2}}^{\eta_{2}}= & \sum_{L=\left|j_{1}-j_{2}\right|}^{j_{1}+j_{2}}(-1)^{M+\eta_{3}} \sqrt{\frac{\left(2 j_{1}+1\right)\left(2 j_{2}+1\right)(2 L+1)}{4 \pi}} \\
& \times\left(\begin{array}{ccc}
j_{1} & j_{2} & L \\
m_{1} & m_{2} & -M
\end{array}\right)\left(\begin{array}{ccc}
j_{1} & j_{2} & L \\
-\eta_{1} & -\eta_{2} & \eta_{3}
\end{array}\right) Y_{L M}^{\eta_{3}}
\end{aligned}
$$

where the Wigner 3- $j$ symbols [47] are used. The values of $M=m_{1}+m_{2}$ and $\eta_{3}=\eta_{1}+\eta_{2}$ are bounded by $-L \leq M \leq L$ and $-L \leq \eta_{3} \leq L$. Substituting the left-hand side of this equation with the definition of spin-weighted spherical harmonics $\mathrm{Y}_{j_{1} m_{1}}^{\eta_{1}}$ and $\mathrm{Y}_{j_{2} m_{2}}^{\eta_{2}}$ from (15) while also assuming that $\eta_{1}=-\eta_{2}=: \eta$, one obtains the relation

$$
\begin{aligned}
\left(\varpi^{\eta} Y_{j_{1} m_{1}}\right)\left(\bar{\partial}^{\eta} Y_{j_{2} m_{2}}\right)= & \sum_{L=\left|j_{1}-j_{2}\right|}^{j_{1}+j_{2}}(-1)^{M+\eta} \sqrt{\frac{\left(2 j_{1}+1\right)\left(2 j_{2}+1\right)(2 L+1)}{4 \pi}} \\
& \times x_{\eta j_{1}}^{j_{2}}\left(\begin{array}{ccc}
j_{1} & j_{2} & L \\
m_{1} & m_{2} & -M
\end{array}\right)\left(\begin{array}{ccc}
j_{1} & j_{2} & L \\
-\eta & \eta & 0
\end{array}\right) \mathrm{Y}_{L M},
\end{aligned}
$$

where the factor $x_{\eta j_{1}}^{j_{2}}$ can be obtained from 15 and is determined by

$$
x_{\eta j_{1}}^{j_{2}}=\sqrt{\frac{\left(j_{2}+\eta\right) !\left(j_{1}+\eta\right) !}{\left(j_{2}-\eta\right) !\left(j_{1}-\eta\right) !}}
$$

Finally, the explicit form of the factor $\kappa$ in 20)-21) is now given by (with $M=m_{1}+m_{2}$ )

$$
\begin{aligned}
{ }^{\eta} \kappa_{j_{1} m_{1}, j_{2} m_{2}}^{L}= & (-1)^{M+\eta} \sqrt{\frac{\left(2 j_{1}+1\right)\left(2 j_{2}+1\right)(2 L+1)}{4 \pi}} \\
& \times x_{|\eta| j_{1}}^{j_{2}}\left(\begin{array}{ccc}
j_{1} & j_{2} & L \\
m_{1} & m_{2} & -M
\end{array}\right)\left(\begin{array}{ccc}
j_{1} & j_{2} & L \\
-\eta & \eta & 0
\end{array}\right) \mathrm{Y}_{L M} .
\end{aligned}
$$




\section{Appendix B. Proof of Result 1}

We prove now Result 1. Both formulas in (32) must satisfy the defining property (14) of the star product. The expansions from (20)-(21) result in the condition

$$
K_{j m, j^{\prime} m^{\prime}}^{L}=\left[\frac{\gamma_{L}}{\gamma_{j} \gamma_{j^{\prime}}}\right]^{ \pm 1} \frac{1}{R} \sum_{\eta=0}^{2 J} \lambda_{\eta}^{( \pm 1) \pm \eta} \kappa_{j_{1} m_{1}, j_{2} m_{2}}^{L}
$$

for $\lambda_{\eta}^{( \pm 1)}$ which holds for every $j, m, j^{\prime}, m^{\prime}$ with $j, j^{\prime} \leq 2 J$. This specifies an overdetermined linear system of equations for $\lambda_{\eta}^{( \pm 1)}$ which can be recognized as the matrix-vector equation $K=\kappa^{( \pm 1)} \lambda^{( \pm 1)}$. Here, the vector $\lambda^{( \pm 1)}$ has the entries $\lambda_{\eta}^{( \pm 1)}$ and every entry $K_{i}$ of the vector $K$ is given by a value of $K_{j_{i} m_{i}, j_{i}^{\prime} m_{i}^{\prime}}^{L_{i}}$ with $i \in\left\{1,2, \ldots,(2 J+1)^{5}\right\}$. The corresponding matrix $\kappa^{( \pm 1)}$ has the dimension $(2 J+1) \times$ $(2 J+1)^{5}$ and rank $(2 J+1)$. This linear system has a unique, exact solution and one obtains the coefficients in 33 .

\section{Appendix C. Asymptotic expansion of weight factors}

Detailed expansion formulas for section 6.2 and section 7.2 are computed in the following. The coefficients in 33 can be expanded into the form

$$
\lambda_{\eta}^{(-1)} \eta !=\frac{(2 J-\eta) !}{(2 J) !}=\prod_{k=0}^{\eta-1}(2 J-k)^{-1}=\prod_{k=0}^{\eta-1}\left[(2 J)^{-1}+k(2 J)^{-2}+\mathcal{O}\left((2 J)^{-3}\right)\right],
$$

where the second equality follows from the Taylor expansion $(a+b)^{-1}=1 / a-b / a^{2}+$ $b^{2} / a^{3}+\cdots$ with $a:=2 J$ and $b:=-k$ and $|b|<a$. Collecting the error terms as $(2 J)^{-\eta+1} \sum_{k=0}^{\eta-1}\left[k(2 J)^{-2}\right]=(2 J)^{-\eta-1}[\eta(\eta-1)] / 2$ yields the formula

$$
\lambda_{\eta}^{(-1)} \eta !=(2 J)^{-\eta}+(2 J)^{-\eta-1}[\eta(\eta-1)]+\mathcal{O}\left((2 J)^{-\eta-2}\right) .
$$

This results in the asymptotic expansion in 34-35. Similarly, $\lambda_{\eta}^{(1)}$ is expanded as

$$
\lambda_{\eta}^{(1)}(-1)^{\eta} \eta !=\frac{2 J(2 J) !}{(2 J+\eta+1) !}=2 J \prod_{k=1}^{\eta+1}(2 J+k)^{-1}=2 J \prod_{k=1}^{\eta+1}\left[(2 J)^{-1}+k(2 J)^{-2}+\mathcal{O}\left((2 J)^{-3}\right)\right],
$$

which simplifies to the asymptotic expansion (which is used in (36))

$$
\lambda_{\eta}^{(1)}(-1)^{\eta} \eta !=(2 J)^{-\eta}+(2 J)^{-\eta-1} \frac{(\eta+1)(\eta+2)}{2}+\mathcal{O}\left((2 J)^{-\eta-2}\right) .
$$

The coefficients in the definition of spin-weighted spherical harmonics in (15) can similarly be expanded as

$$
\sqrt{(j-\eta) ! /(j+\eta) !}=\prod_{k=-\eta+1}^{\eta}(j+k)^{1 / 2}=\prod_{k=-\eta+1}^{\eta}\left[j^{1 / 2}+\frac{k}{2 j^{1 / 2}}+\mathcal{O}\left(j^{-3 / 2}\right)\right]
$$

where the second equality is obtained from the Taylor expansion $(a+b)^{1 / 2}=a^{1 / 2}+$ $b /\left(2 a^{1 / 2}\right)-b^{2} /\left(8 a^{3 / 2}\right)+\cdots$ with $a:=j$ and $b:=k$ and $b<a$. This yields the expansion

$$
\sqrt{(j-\eta) ! /(j+\eta) !}=j^{\eta}+\frac{\eta}{2 j^{1 / 2}}+\mathcal{O}\left(j^{-5 / 2}\right) \text {. }
$$


Following similar arguments, the factor $\gamma_{j}$, which is defined in (11), can be in terms of $j(j+1)$ expanded into the exponential function

$$
\gamma_{j}=\exp [-j(j+1) /(4 J)]+\mathcal{O}\left(J^{-1}\right)=\sum_{n}[-j(j+1) /(4 J)]^{n} / n !+\mathcal{O}\left(J^{-1}\right) .
$$

This expansion is used in (43) to derive an approximation of the operator $\square(s)$.

\section{Appendix D. Proof of Result 3 and the associated expansion coefficients}

We now prove Result 3 and determine the corresponding expansion coefficients $c_{n}(s)$. The coefficients $c_{n}(s)$ are uniquely determined by the values of $\gamma_{j}^{1-s}$ and the condition

$$
\gamma_{j}^{1-s}=\sum_{n=0}^{2 J} c_{n}(s)[-j(j+1)]^{n} \text { for } 0 \leq j \leq 2 J .
$$

This yields the linear system $V c(s)=\gamma(s)$ of equations where $V$ is the Vandermonde matrix with entries $[V]_{n j}:=[-j(j+1)]^{n}$ and its inverse $V^{-1}$ can be computed analytically [70]. The entries of the vectors $c(s)$ and $\gamma(s)$ are given by $c_{n}(s)$ and $\gamma_{j}^{1-s}$, respectively. The exact, unique solution is determined by $V^{-1} \gamma(s)=c(s)$.

Simultaneously truncating the spherical-harmonics decomposition can also be achieved by enlarging the summation upper limit in (D.1) to $4 J$. In that case, one has $\square(s) \mathrm{Y}_{j m}=0$ for $2 J<j \leq 4 J$. Alternatively, a projection operator $\mathcal{P}_{J}$ from Result 2 of 34] can be applied to spin- $J$ phase-space representations, where $\mathcal{P}_{J}:=\sum_{n=0}^{4 J} p_{n}(\delta \bar{\delta})^{n}$ and the coefficients $p_{n}$ are computed from the linear system of equations

$$
\sum_{n=0}^{4 J} p_{n}[-j(j+1)]^{n}= \begin{cases}1 & \text { for } 0 \leq j \leq 2 J \\ 0 & \text { for } 2 J<j \leq 4 J\end{cases}
$$

which is determined by the inverse Vandermonde matrix $V^{-1}$.

\section{Appendix E. Asymptotic expansion of differential operators}

\section{Appendix E.1. Expansion formulas using polar and arc-length parametrizations}

In this section, we show how the operators $\partial$ and $\bar{\partial}$ approach their infinite-dimensional counterparts given by the derivatives $\partial_{\alpha^{*}}$ and $\partial_{\alpha}$. We consider the polar parametrization $\alpha=r e^{i \phi}$ of the complex plane with $r=\sqrt{\alpha^{*} \alpha}$ and $\phi:=\arg \alpha$. Using $\partial_{\alpha}=\frac{\partial r}{\partial \alpha} \partial_{r}+\frac{\partial \phi}{\partial \alpha} \partial_{\phi}$, the derivatives $\partial_{\alpha}$ and $\partial_{\alpha^{*}}$ can be expressed in the polar parametrization by substituting $\frac{\partial r}{\partial \alpha}=\frac{1}{2} e^{-i \phi}$ and $\frac{\partial \phi}{\partial \alpha}=\frac{-i}{2} e^{-i \phi} / r$ which results in

$$
\partial_{\alpha}=e^{-i \phi} \frac{1}{2}\left[\partial_{r}-i / r \partial_{\phi}\right] \text { and } \partial_{\alpha^{*}}=e^{i \phi} \frac{1}{2}\left[\partial_{r}+i / r \partial_{\phi}\right] .
$$

Applying these formulas one obtains formulas for powers of derivatives:

$$
\left[\partial_{\alpha^{*}}\right]^{\eta}=e^{i \eta \phi} \prod_{k=0}^{\eta-1} \frac{1}{2}\left[-k / r+\partial_{r}+i / r \partial_{\phi}\right],\left[\partial_{\alpha}\right]^{\eta}=e^{-i \eta \phi} \prod_{k=0}^{\eta-1} \frac{1}{2}\left[-k / r+\partial_{r}-i / r \partial_{\phi}\right] .
$$


For comparison, we apply the spin-weight raising and lowering differential operators from (17) and (18) and obtain

$$
\begin{aligned}
& {[\check{\mathrm{d}} / \sqrt{2 J}]^{\eta} \mathrm{Y}_{j m}=(-1)^{\eta} \prod_{k=0}^{\eta-1} \frac{1}{2}\left(-k \frac{\cos \theta}{\sqrt{J / 2} \sin \theta}+\frac{\partial_{\theta}}{\sqrt{J / 2}}+\frac{i}{\sqrt{J / 2} \sin \theta} \partial_{\phi}\right) \mathrm{Y}_{j m}} \\
& {[\overline{\widetilde{\partial}} / \sqrt{2 J}]^{\eta} \mathrm{Y}_{j m}=(-1)^{\eta} \prod_{k=0}^{\eta-1} \frac{1}{2}\left(-k \frac{\cos \theta}{\sqrt{J / 2} \sin \theta}+\frac{\partial_{\theta}}{\sqrt{J / 2}}-\frac{i}{\sqrt{J / 2} \sin \theta} \partial_{\phi}\right) \mathrm{Y}_{j m} .}
\end{aligned}
$$

The arc-length parametrization $\alpha=\sqrt{J / 2} \theta e^{-i \phi}$ (see, e.g., 28 ) implies that $r=$ $\sqrt{\alpha^{*} \alpha}=\sqrt{J / 2} \theta$. The following terms from $($ E.3) and E.4) can be expanded by applying their Taylor series and substituting $\theta=r / \sqrt{J / 2}$ :

$$
\frac{i}{\sqrt{J / 2} \sin (r / \sqrt{J / 2})}=\frac{i}{r}+\frac{i r}{3 J}+\mathcal{O}\left(J^{-3 / 2}\right) \text { and } \frac{\cos (r / \sqrt{J / 2})}{\sqrt{J / 2} \sin (r / \sqrt{J / 2})}=\frac{1}{r}-\frac{2 r}{3 J}+\mathcal{O}\left(J^{-3 / 2}\right) .
$$

Substituting these expansions back into E.3 and E.4 results in

$$
\begin{aligned}
& {[ð / \sqrt{2 J}]^{\eta} \mathrm{Y}_{j m}=(-1)^{\eta} \prod_{k=0}^{\eta-1} \frac{1}{2}\left[-k\left(\frac{1}{r}-\frac{2 r}{3 J}\right)+\partial_{r}+\left(\frac{i}{r}+\frac{i r}{3 J}\right) \partial_{\phi}+\mathcal{O}\left(J^{-3 / 2}\right)\right] \mathrm{Y}_{j m},} \\
& {[\overline{\mathrm{\partial}} / \sqrt{2 J}]^{\eta} \mathrm{Y}_{j m}=(-1)^{\eta} \prod_{k=0}^{\eta-1} \frac{1}{2}\left[-k\left(\frac{1}{r}-\frac{2 r}{3 J}\right)+\partial_{r}-\left(\frac{i}{r}+\frac{i r}{3 J}\right) \partial_{\phi}+\mathcal{O}\left(J^{-3 / 2}\right)\right] \mathrm{Y}_{j m} .}
\end{aligned}
$$

Note that $\partial_{\phi} \mathrm{Y}_{j m}^{\eta}=i m \mathrm{Y}_{j m}^{\eta}$. The expressions in the parentheses can up to an error term $\epsilon:=\frac{r}{3 J}(2 k-m)$ be transformed into terms that are directly comparable to (E.2):

$$
\begin{aligned}
& {[ð / \sqrt{2 J}]^{\eta} \mathrm{Y}_{j m}=(-1)^{\eta} \prod_{k=0}^{\eta-1} \frac{1}{2}\left[-k / r+\partial_{r}+i / r \partial_{\phi}+\epsilon+\mathcal{O}\left(J^{-3 / 2}\right)\right] \mathrm{Y}_{j m},} \\
& {[\overline{\widetilde{\partial}} / \sqrt{2 J}]^{\eta} \mathrm{Y}_{j m}=(-1)^{\eta} \prod_{k=0}^{\eta-1} \frac{1}{2}\left[-k / r+\partial_{r}-i / r \partial_{\phi}-\epsilon+\mathcal{O}\left(J^{-3 / 2}\right)\right] \mathrm{Y}_{j m} .}
\end{aligned}
$$

We compare E.2 with E.6 and E.7), apply $\sum_{k=0}^{\eta-1} \epsilon / 2=\frac{r}{6 J}(1+\eta)(\eta-m)$, and denote the residual error terms by $\zeta:=\mathcal{\mathcal { O }}\left(J^{-3 / 2}[ð / \sqrt{2 J}]^{\eta-1}\right)$ and $\bar{\zeta}:=\mathcal{O}\left(J^{-3 / 2}[\overline{\widetilde{\delta}} / \sqrt{2 J}]^{\eta-1}\right)$. This leads to

$$
\begin{aligned}
& {[\check{\delta} / \sqrt{2 J}]^{\eta} \mathrm{Y}_{j m}=\left[(-1)^{\eta} e^{-i \eta \phi}\left(\partial_{\alpha^{*}}\right)^{\eta}+\frac{r(1+\eta)(\eta-m)}{6 J}[ð / \sqrt{2 J}]^{\eta-1}+\zeta\right] \mathrm{Y}_{j m}} \\
& {[\overline{\widetilde{\partial}} / \sqrt{2 J}]^{\eta} \mathrm{Y}_{j m}=\left[(-1)^{\eta} e^{i \eta \phi}\left(\partial_{\alpha}\right)^{\eta}-\frac{r(1+\eta)(\eta-m)}{6 J}[\overline{\widetilde{\partial}} / \sqrt{2 J}]^{\eta-1}+\bar{\zeta}\right] \mathrm{Y}_{j m} .}
\end{aligned}
$$

Substituting this expansion into the definition of spin-weighted spherical harmonics in (15), one obtains for a fixed arc length $\alpha$ the forms (refer to (22)-(23) and Figure $3(\mathrm{a}-\mathrm{b})$ )

$$
\begin{aligned}
& \mathrm{Y}_{j m}^{\eta}-(-1)^{\eta} e^{-i \eta \phi}\left(\partial_{\alpha^{*}}\right)^{\eta} \mathrm{Y}_{j m} \propto|\alpha|(j \sqrt{J})^{-1} \mathrm{Y}_{j m}^{\eta-1}+\mathcal{O}\left(J^{-1}\right) \text { and } \\
& \mathrm{Y}_{j m}^{-\eta}-e^{i \eta \phi}\left(\partial_{\alpha}\right)^{\eta} \mathrm{Y}_{j m} \propto|\alpha|(j \sqrt{J})^{-1} \mathrm{Y}_{j m}^{-\eta+1}+\mathcal{O}\left(J^{-1}\right) .
\end{aligned}
$$

The difference on the left-hand side vanishes in the limit of infinite $J$ for every bounded $\alpha$, as the spin-weighted spherical harmonics $\mathrm{Y}_{j m}^{-\eta \pm 1}$ on the right-hand side are bounded, i.e., $\left|\mathrm{Y}_{j m}^{-\eta \pm 1}(\theta, \phi)\right|<\infty$.

We now describe a general criterion (see section 5) for spherical functions and their differentials to be bounded. Assume now that the spherical function $f=f(\theta, \phi)=$ 
$\sum f_{j m} \mathrm{Y}_{j m}(\theta, \phi)$ is bounded, i.e., $|f(\theta, \phi)|<\infty$. Note that the expansion coefficients might depend on $J$. Also, assume that the differentials are bounded, i.e., ||$^{\eta} f(\theta, \phi) \mid<\infty$ and $\left|\bar{\partial}^{\eta} f(\theta, \phi)\right|<\infty$, which translates to

$$
\begin{aligned}
& \left|(ð / \sqrt{2 J})^{\eta} f(\theta, \phi)\right|=\left|\sum{\sqrt{(2 J)^{\eta}(j-\eta) ! /(j+\eta) !}}^{-1} f_{j m} Y_{j m}^{\eta}(\theta, \phi)\right|<\infty \\
& \left|(\bar{\delta} / \sqrt{2 J})^{\eta} f(\theta, \phi)\right|=\left|\sum{\sqrt{(2 J)^{\eta}(j-\eta) ! /(j+\eta) !}}^{-1} f_{j m} Y_{j m}^{-\eta}(\theta, \phi)\right|<\infty .
\end{aligned}
$$

We emphasize that $f$ and all of its derivatives are bounded if there are only a finite number of non-zero expansion coefficients $f_{j m}$ or if the expansion coefficients $\left|f_{j m}\right|$ decay faster in $j$ than the coefficients $\sqrt{(j-\eta) ! /(j+\eta) !} \approx j^{-\eta}$ from (C.1). Applying (E.6) and E.7) to the spherical function $f$ one gets for a fixed arc length $|\alpha|$ that

$$
\begin{aligned}
& {\left[(ð / \sqrt{2 J})^{\eta}-(-1)^{\eta} e^{-i \eta \phi}\left(\partial_{\alpha^{*}}\right)^{\eta}\right] f(\alpha) \propto|\alpha| J^{-1}[\check{\delta} / \sqrt{2 J}]^{\eta-1} f(\alpha)} \\
& {\left[(\bar{\delta} / \sqrt{2 J})^{\eta}-(-1)^{\eta} e^{i \eta \phi}\left(\partial_{\alpha}\right)^{\eta}\right] f(\alpha) \propto|\alpha| J^{-1}[\bar{\delta} / \sqrt{2 J}]^{\eta-1} f(\alpha) .}
\end{aligned}
$$

This difference clearly vanishes if $g=|\alpha|[\partial / \sqrt{2 J}]^{\eta-1} f(\alpha)$ remains bounded in the limit of infinite $J$. (The assumption could be weakened such that the growth of the absolute value of $g$ in $J$ is slower than $\mathcal{O}(J)$.) For a fixed $\alpha$, this expansion of the action of spin-weight lowering and raising operators has the convergence rate $\mathcal{O}\left(J^{-1}\right)$, refer to Proposition 1.

We now describe when spherical functions and their differentials are in general bounded in the $L^{2}$ norm, and this information is utilized in section 5. The asymptotic behavior of the difference function can be measured in the $L^{2}$ norm. Assume that the square-integrable spherical function $f=f(\theta, \phi)=\sum f_{j m} Y_{j m}(\theta, \phi)$ observes $R^{2} \sum\left|f_{j m}\right|^{2}=1$. Also asssume that its differentials are square integrable. Applying the orthonormality of spin-weighted spherical harmonics, this translates to

$$
\begin{aligned}
& \|\left((\bar{\delta} / \sqrt{2 J})^{\eta} f(\theta, \phi) \|_{L^{2}}=R^{2} \sum\left((2 J)^{\eta}(j-\eta) ! /(j+\eta) !\right)^{-1}\left|f_{j m}\right|^{2}<\infty,\right. \\
& \left\|(\bar{\delta} / \sqrt{2 J})^{\eta} f(\theta, \phi)\right\|_{L^{2}}=R^{2} \sum\left((2 J)^{\eta}(j-\eta) ! /(j+\eta) !\right)^{-1}\left|f_{j m}\right|^{2}<\infty .
\end{aligned}
$$

Note that $f$ and all of its derivatives are square integrable for finite $J$ if there are only a finite number of non-zero expansion coefficients $f_{j m}$ or if the expansion coefficients $\left|f_{j m}\right|^{2}$ decay faster in $j$ than $(j-\eta) ! /(j+\eta) ! \approx j^{-2 \eta}$ from (C.1). Applying (E.6) and (E.7), the norm of the difference is given by

$$
\begin{aligned}
& \left\|\left[(\widetilde{\delta} / \sqrt{2 J})^{\eta}-(-1)^{\eta} e^{-i \eta \phi}\left(\partial_{\alpha^{*}}\right)^{\eta}\right] f(\theta, \phi)\right\|_{L^{2}} \propto J^{-1}\left\||\alpha|[\check{[} / \sqrt{2 J}]^{\eta-1} f(\theta, \phi)\right\|_{L^{2}} \\
& \left\|\left[(\bar{\delta} / \sqrt{2 J})^{\eta}-(-1)^{\eta} e^{i \eta \phi}\left(\partial_{\alpha}\right)^{\eta}\right] f(\theta, \phi)\right\|_{L^{2}} \propto J^{-1}\left\||\alpha|[\bar{\delta} / \sqrt{2 J}]^{\eta-1} f(\theta, \phi)\right\|_{L^{2}} .
\end{aligned}
$$

This difference clearly vanishes if the norm $\left\||\alpha|[\widetilde{\delta} / \sqrt{2 J}]^{\eta-1} f(\theta, \phi)\right\|_{L^{2}}$ remains bounded in the large-spin limit. (This assumption can be weakened such that the growth of this norm in $J$ is slower than $\mathcal{O}(J)$.) Refer to Proposition 1.

Alternatively, the following expansions can be derived from (E.6) and (E.7):

$$
\begin{aligned}
& {\left[(\bar{\delta} / \sqrt{2 J})^{\eta}-(-1)^{\eta} e^{-i \eta \phi}\left(\partial_{\alpha^{*}}\right)^{\eta}\right] f(\alpha) \propto J^{-1}|\alpha| \frac{\partial^{\eta-1} f(\alpha)}{\left(\partial_{\alpha^{*}}\right)^{\eta-1}},} \\
& {\left[(\bar{\delta} / \sqrt{2 J})^{\eta}-(-1)^{\eta} e^{i \eta \phi}\left(\partial_{\alpha}\right)^{\eta}\right] f(\alpha) \propto J^{-1}|\alpha| \frac{\partial^{\eta-1} f(\alpha)}{\left(\partial_{\alpha}\right)^{\eta-1}} .}
\end{aligned}
$$


These differences vanish in the limit of infinite $J$ if the derivatives remain bounded, i.e.,

$$
|| \alpha\left|\frac{\partial^{\eta-1} f\left(\alpha, \alpha^{*}\right)}{\left(\partial_{\alpha^{*}}\right)^{\eta-1}}\right|<\infty \text { and }|| \alpha\left|\frac{\partial^{\eta-1} f\left(\alpha, \alpha^{*}\right)}{\left(\partial_{\alpha^{*}}\right)^{\eta-1}}\right|<\infty .
$$

In addition, the $L^{2}$ norm of the differences vanishes if the derivatives remain square integrable, i.e.,

$$
\left\||\alpha| \frac{\partial^{\eta-1} f\left(\alpha, \alpha^{*}\right)}{\left(\partial_{\alpha^{*}}\right)^{\eta-1}}\right\|_{L^{2}}<\infty \text { and }\left\||\alpha| \frac{\partial^{\eta-1} f\left(\alpha, \alpha^{*}\right)}{\left(\partial_{\alpha^{*}}\right)^{\eta-1}}\right\|_{L^{2}}<\infty
$$

or if the growth of the norm and the absolute value in $J$ is slower than $\mathcal{O}(J)$. This is used in Proposition 1 .

Following similar arguments, asymptotic expansions for products of differentials from Proposition 2 are obtained in the formulas

$$
\begin{aligned}
& \left|f\left[\left(\overleftarrow{\partial}^{\eta}\right)\left(\overrightarrow{\bar{\partial}}^{\eta}\right) /(2 J)^{\eta}\right] g-f\left(\overleftarrow{\partial}_{\alpha^{*}}\right)^{\eta}\left(\vec{\partial}_{\alpha}\right)^{\eta} g\right| \propto J^{-1} \text { and } \\
& \left\|f\left[\left(\overleftarrow{\check{\partial}^{\eta}}\right)\left(\overrightarrow{\bar{\partial}}^{\eta}\right) /(2 J)^{\eta}-\left(\overleftarrow{\partial}_{\alpha^{*}}\right)^{\eta}\left(\vec{\partial}_{\alpha}\right)^{\eta}\right] g\right\|_{L^{2}} \propto J^{-1}
\end{aligned}
$$

and the two formulas are also valid for the conjugate derivatives. Consider two squareintegrable functions $f$ and $g$ with the additional constraint that the product $f g$ as well as the products of differentials $|\alpha|\left(\partial^{\eta} f\right)\left(\overline{\widetilde{\partial}}^{\eta} g\right)$ are square integrable. The $L^{2}$-norm convergence then holds (refer to Proposition 2).

\section{Appendix E.2. The product $\precsim \bar{\partial}$ of the spin-weight raising and lowering operators}

We now derive the second part of Proposition 1. The derivatives $\partial_{\alpha^{*}} \partial_{\alpha}$ in the polar parametrization are expanded into

$$
\partial_{\alpha^{*}} \partial_{\alpha}=\left[-1 / r+\partial_{r}+i / r \partial_{\phi}\right]\left[\partial_{r}-i / r \partial_{\phi}\right] / 4 \text {. }
$$

Similarly, the expansion of the operator $ð \bar{\gamma} /(2 J)$ is given by

$$
ð \bar{\partial} /(2 J) \mathrm{Y}_{j m}=\left[-\frac{\cos \theta}{\sqrt{2 J} \sin \theta}+\partial_{\theta} / \sqrt{2 J}+\frac{i}{\sqrt{2 J} \sin \theta} \partial_{\phi}\right]\left[\partial_{\theta} / \sqrt{2 J}-\frac{i}{\sqrt{2 J} \sin \theta} \partial_{\phi}\right] \mathrm{Y}_{j m}
$$

Applying the expansions from (E.5) and the parametrization $\theta=r / \sqrt{J / 2}$ yields

$$
\begin{aligned}
\precsim \bar{\partial} /(2 J) \mathrm{Y}_{j m}= & {\left[-\frac{1}{r}-\frac{2 r}{3 J}+\partial_{r}+\left(\frac{i}{r}+\frac{i r}{3 J}\right) \partial_{\phi}+\mathcal{O}\left(J^{-3 / 2}\right)\right] } \\
& \times\left[\partial_{r}-\left(\frac{i}{r}+\frac{i r}{3 J}\right) \partial_{\phi}+\mathcal{O}\left(J^{-3 / 2}\right)\right] \mathrm{Y}_{j m} / 4
\end{aligned}
$$

Now separating the terms and expanding the action $\partial_{\phi} \mathrm{Y}_{j m}$ results in

$$
\begin{aligned}
\precsim \bar{\varnothing} /(2 J) \mathrm{Y}_{j m}=[ & \left.-\frac{1}{r}+\partial_{r}+\frac{i}{r} \partial_{\phi}-\frac{(m+2) r}{3 J}+\mathcal{O}\left(J^{-3 / 2}\right)\right] \\
& \times\left[\partial_{r}-\frac{i}{r} \partial_{\phi}-\frac{m r}{3 J}+\mathcal{O}\left(J^{-3 / 2}\right)\right] \mathrm{Y}_{j m} / 4
\end{aligned}
$$

Finally, we obtain for a bounded spherical function $f$ that

$$
\left[ð \bar{\varnothing} /(2 J)-\partial_{\alpha^{*}} \partial_{\alpha}\right] f \propto|\alpha| J^{-1} \text { and }\left\|\left[ð \overline{\widetilde{\partial}} /(2 J)-\partial_{\alpha^{*}} \partial_{\alpha}\right] f\right\|_{L^{2}} \propto|\alpha| J^{-1} \text {. }
$$

The norm or the absolute value vanish in the large-spin limit if both the function $f$ and its differentials are bounded or square integrable in the limit, see Proposition 1. 


\section{Appendix F. Proof of Result 6}

We prove Result 6. Substituting the approximations of $\square(s)$ from $\sqrt{44}$ ) and of $\star^{(-1)}$ form (39) into 46) yields the formula

$$
\begin{aligned}
& f\left[\overleftarrow{\square}(-s) \star^{(-1)} \vec{\square}_{(-s)] g}\right. \\
& =f \exp \left[\frac{1+s}{2} \overleftarrow{\partial}_{\alpha^{*}} \overleftarrow{\partial}_{\alpha}\right] \exp \left[\overleftarrow{\partial}_{\alpha} \vec{\partial}_{\alpha^{*}}\right] \exp \left[\frac{1+s}{2} \vec{\partial}_{\alpha^{*}} \vec{\partial}_{\alpha}\right] g+\mathcal{O}\left(J^{-1}\right) \\
& =f \exp \left[\frac{1+s}{2} \overleftarrow{\partial}_{\alpha^{*}} \overleftarrow{\partial}_{\alpha}+\overleftarrow{\partial}_{\alpha} \vec{\partial}_{\alpha^{*}}+\frac{1+s}{2} \vec{\partial}_{\alpha^{*}} \vec{\partial}_{\alpha}\right] g+\mathcal{O}\left(J^{-1}\right)
\end{aligned}
$$

where the second equality follows from the commutativity of partial derivatives. Using the Leibniz rule of partial derivatives

$$
\partial_{\alpha^{*}} \partial_{\alpha}(f g)=\left(\partial_{\alpha^{*}} \partial_{\alpha} f\right) g+f\left(\partial_{\alpha^{*}} \partial_{\alpha} g\right)+\left(\partial_{\alpha^{*}} f\right)\left(\partial_{\alpha} g\right)+\left(\partial_{\alpha} f\right)\left(\partial_{\alpha^{*}} g\right)
$$

results in a convenient description for the action of the approximation of $\square(s) f g$ :

$$
\exp \left[-\frac{1+s}{2} \partial_{\alpha^{*}} \partial_{\alpha}\right] f g=f \exp \left[-\frac{1+s}{2}\left(\overleftarrow{\partial}_{\alpha^{*}} \overleftarrow{\partial}_{\alpha}+\vec{\partial}_{\alpha^{*}} \vec{\partial}_{\alpha}+\overleftarrow{\partial}_{\alpha^{*}} \vec{\partial}_{\alpha}+\overleftarrow{\partial}_{\alpha} \vec{\partial}_{\alpha^{*}}\right)\right] g
$$

Substituting this into $\square(s+2)\left(f\left[\overleftarrow{\square}(-s) \star^{(-1)} \vec{\square}(-s)\right] g\right)$, one obtains 52 which is expanded as

$$
f \star^{(s)} g=\sum_{n=0}^{\infty} \sum_{m=0}^{n} c_{n m}(s)\left(\partial_{\alpha}^{m} \partial_{\alpha^{*}}^{n-m} f\right)\left(\partial_{\alpha^{*}}^{m} \partial_{\alpha}^{n-m} g\right)+\mathcal{O}\left(J^{-1}\right),
$$

where $c_{n m}(s)$ are the expansion coefficients of the exponential $\exp \left[\frac{(1-s)}{2} a-\frac{(1+s)}{2} b\right]=$ $\sum_{n=0}^{\infty} \sum_{m=0}^{n} c_{n m}(s) a^{m} b^{n-m}$ for commutative $a$ and $b$. Using the polar parametrization from $(\mathrm{E} .2)$, the derivatives $\partial_{\alpha}^{n} \partial_{\alpha^{*}}^{m}$ can be represented as

$$
\partial_{\alpha}^{n} \partial_{\alpha^{*}}^{m}=e^{i(m-n) \phi} \prod_{\eta=-m}^{n-m-1} \frac{1}{2}\left[-\eta / r+\partial_{r}-i / r \partial_{\phi}\right] \prod_{\eta=0}^{m-1} \frac{1}{2}\left[-\eta / r+\partial_{r}+i / r \partial_{\phi}\right] .
$$

One applies arguments from Appendix E and the expansion

$$
\left(\partial_{\alpha}^{n} \partial_{\alpha^{*}}^{m} f\right)\left(\partial_{\alpha^{*}}^{n} \partial_{\alpha}^{m} g\right)=(2 J)^{-n-m}\left(\bar{\partial}^{n} \check{\partial}^{m} f\right)\left(\check{\partial}^{n} \bar{\partial}^{m} g\right)+\mathcal{O}\left(J^{-1}\right)
$$

of the differential operators can be established which finally yields (51).

\section{Appendix G. Details for the example in section 10}

We discuss some details for the example in section 10. The normalization factor $N$ in 69. can be computed using $1 / N^{2}=\left\langle J J\left|K^{\dagger} K\right| J J\right\rangle$ where

$$
\begin{aligned}
K & =\mathcal{R}^{-1}\left(\Omega_{0}\right) \mathcal{J}_{-} \mathcal{R}\left(\Omega_{0}\right) / \sqrt{2 J} \\
& =\left[\mathcal{J}_{-} D_{-1,-1}^{1}\left(\Omega^{\prime}\right)+\mathcal{J}_{z} D_{0,-1}^{1}\left(\Omega^{\prime}\right) / \sqrt{2}+\mathcal{J}_{+} D_{1,-1}^{1}\left(\Omega^{\prime}\right)\right] / \sqrt{2 J}
\end{aligned}
$$

Here, $D_{m, m^{\prime}}^{j}$ are Wigner D-matrix elements 1]. All the contributions in (G.1) vanish except for $\left\langle J J\left|\mathcal{J}_{+} \mathcal{J}_{-}\right| J J\right\rangle=2 J$ and $\left\langle J J\left|\mathcal{J}_{z} \mathcal{J}_{z}\right| J J\right\rangle=J^{2}$. Finally, one obtains $1 / N^{2}=\cos (\theta / 2)^{2}[1+2 J-(2 J-1) \cos (\theta)] / 2$. 
The phase-space representation $F_{K\left(\Omega_{0}\right)}$ of the operator $K$ from 69 can be specified in terms of spherical harmonics as 34

$$
\begin{aligned}
& F_{K\left(\Omega_{0}\right)}=c_{s} \mathcal{R}\left(\Omega_{0}\right) \mathrm{Y}_{1,-1}(\Omega) \\
& =c_{s}\left[\mathrm{Y}_{1,-1}(\Omega) D_{-1,-1}^{1}\left(\Omega_{0}\right)+\mathrm{Y}_{1,0}(\Omega) D_{0,-1}^{1}\left(\Omega_{0}\right)+\mathrm{Y}_{1,1}(\Omega) D_{1,-1}^{1}\left(\Omega_{0}\right)\right]
\end{aligned}
$$

where the rotation can be written in terms of Wigner D-matrices and the prefactor is given by $c_{s}=N \sqrt{(J+1)(2 J+1) / 3} \gamma_{1}^{-s} / R$.

The star product with $F_{K\left(\Omega_{0}\right)}$ in $(70)$ and $(71)$ can be approximated using (51). The approximate actions of $\mathcal{K}\left(\Omega_{0}\right)$ and $\overline{\mathcal{K}}\left(\Omega_{0}\right)$ are then given by

$$
\begin{aligned}
& \mathcal{K}\left(\Omega_{0}\right) f=\left[F_{K\left(\Omega_{0}\right)}+\frac{1-s}{4 J}\left(\overline{\widetilde{\partial}} F_{K\left(\Omega_{0}\right)}\right) \partial-\frac{1+s}{4 J}\left(ð F_{K\left(\Omega_{0}\right)}\right) \bar{\varnothing}\right] f+\mathcal{O}\left(J^{-1}\right) \\
& \overline{\mathcal{K}}\left(\Omega_{0}\right) f=\left[\left(F_{K\left(\Omega_{0}\right)}\right)^{*}+\frac{1-s}{4 J}\left(ð\left(F_{K\left(\Omega_{0}\right)}\right)^{*}\right) \bar{ð}-\frac{1+s}{4 J}\left(\bar{ð}\left(F_{K\left(\Omega_{0}\right)}\right)^{*}\right) ð\right] f+\mathcal{O}\left(J^{-1}\right) .
\end{aligned}
$$

Using the star-product approximation from $(52)$, the actions of $\mathcal{K}\left(\Omega_{0}\right)$ and $\overline{\mathcal{K}}\left(\Omega_{0}\right)$ can be expanded into

$$
\begin{aligned}
& \mathcal{K}\left(\Omega_{0}\right) f=\left[F_{K\left(\Omega_{0}\right)}+\frac{1-s}{4 J}\left(\partial_{\alpha} F_{K\left(\Omega_{0}\right)}\right) \partial_{\alpha^{*}}-\frac{1+s}{4 J}\left(\partial_{\alpha^{*}} F_{K\left(\Omega_{0}\right)}\right) \partial_{\alpha}\right] f+\mathcal{O}\left(J^{-1}\right) \\
& \overline{\mathcal{K}}\left(\Omega_{0}\right) f=\left[\left(F_{K\left(\Omega_{0}\right)}\right)^{*}+\frac{1-s}{4 J}\left(\partial_{\alpha^{*}}\left(F_{K\left(\Omega_{0}\right)}\right)^{*}\right) \partial_{\alpha}-\frac{1+s}{4 J}\left(\partial_{\alpha}\left(F_{K\left(\Omega_{0}\right)}\right)^{*}\right) \partial_{\alpha^{*}}\right] f+\mathcal{O}\left(J^{-1}\right) .
\end{aligned}
$$

Knowing that $F_{K(0)}=c_{s} \mathrm{Y}_{1,-1}(\Omega)$ with $\check{\partial Y_{1,-1}}=\sqrt{2} \mathrm{Y}_{1,-1}^{1}, \overline{\widehat{\partial}} \mathrm{Y}_{1,-1}=-\sqrt{2} \mathrm{Y}_{1,-1}^{-1}$, and $\left(\mathrm{Y}_{1,-1}\right)^{*}=\mathrm{Y}_{1,1}$, the action of $\mathcal{K}\left(\Omega_{0}\right)$ and $\overline{\mathcal{K}}\left(\Omega_{0}\right)$ at the point $\Omega_{0}=0$ is given by

$$
\begin{aligned}
& \mathcal{K}(0) f=\mathcal{K} f=c_{s}\left[\mathrm{Y}_{1,-1}-\sqrt{2} \frac{1-s}{4 J} \mathrm{Y}_{1,-1}^{-1} \mathrm{\partial}-\sqrt{2} \frac{1+s}{4 J} \mathrm{Y}_{1,-1}^{1} \overline{\mathrm{\jmath}}\right] f+\mathcal{O}\left(J^{-1}\right) \text { and } \\
& \overline{\mathcal{K}}(0) f=\overline{\mathcal{K}} f=c_{s}\left[\mathrm{Y}_{1,1}+\sqrt{2} \frac{1-s}{4 J} \mathrm{Y}_{1,1}^{1} \overline{\bar{\partial}}+\sqrt{2} \frac{1+s}{4 J} \mathrm{Y}_{1,1}^{-1} \mathrm{\partial}\right] f+\mathcal{O}\left(J^{-1}\right),
\end{aligned}
$$

which are then used in $73(74)$.

\section{References}

[1] Cohen-Tannoudji C, Diu B and Laloe F 1991 Quantum Mechanics, Vol. 1 (Wiley, New York)

[2] Feynman R P and Hibbs A R 1965 Quantum Mechanics and Path Integrals (McGraw-Hill, New York)

[3] Leonhardt U 1997 Measuring the Quantum State of Light (Cambridge Univ. Press, Cambridge)

[4] Carruthers P and Zachariasen F 1983 Rev. Mod. Phys. 55245

[5] Hillery M, O'Connell R F, Scully M O and Wigner E P 1984 Phys. Rep. 106 121-167

[6] Kim Y S and Noz M E 1991 Phase Space Picture of Quantum Mechanics: Group Theoretical Approach (World Scientific, Singapore)

[7] Lee H W 1995 Phys. Rep. 259 147-211

[8] Gadella M 1995 Fortschr. Phys. 43229

[9] Zachos C K, Fairlie D B and Curtright T L 2005 Quantum Mechanics in Phase Space: An Overview with Selected Papers (World Scientific, Singapore)

[10] Schroeck Jr F E 2013 Quantum Mechanics on Phase Space (Springer, Dordrecht)

[11] Schleich W P 2001 Quantum Optics in Phase Space (Wiley-VCH, Berlin)

[12] Curtright T L, Fairlie D B and Zachos C K 2014 A Concise Treatise on Quantum Mechanics in Phase Space (World Scientific, Singapore)

[13] Wigner E 1932 Phys. Rev. 40749

[14] Husimi K 1940 Proc. Phys. Math. Soc. Japan 22 264-314

[15] Cahill K E and Glauber R 1969 Phys. Rev. 1771882

[16] Stratonovich R L 1956 J. Exptl. Theoret. Phys. (U.S.S.R.) 31 1012-1020 
[17] Agarwal G S 1981 Phys. Rev. A 24 2889-2896

[18] Dowling J P, Agarwal G S and Schleich W P 1994 Phys. Rev. A 49 4101-4109

[19] Várilly J C and Garcia-Bondía J M 1989 Ann. Phys. 190 107-148

[20] Brif C and Mann A 1997 J. Phys. A 31 L9-L17

[21] Brif C and Mann A 1999 Phys. Rev. A 59971

[22] Heiss S and Weigert S 2000 Phys. Rev. A 63012105

[23] Klimov A B and Espinoza P 2002 J. Phys. A 358435

[24] Klimov A 2002 J. Math. Phys. 43 2202-2213

[25] Klimov A and Espinoza P 2005 J. Opt. B 7183

[26] Klimov A and Romero J 2008 J. Phys. A 41055303

[27] Klimov A B and de Guise H 2010 J. Phys. A 43402001

[28] Koczor B, Zeier R and Glaser S J 2017 Continuous phase-space representations for finitedimensional quantum states and their tomography (Preprint arXiv:1711.07994v2)

[29] Tilma T, Everitt M J, Samson J H, Munro W J and Nemoto K 2016 Phys. Rev. Lett. 117180401

[30] Philp D J and Kuchel P W 2005 Concepts Magn. Reso. A 25A 40-52

[31] Merkel S T, Jessen P S and Deutsch I H 2008 Phys. Rev. A 78023404

[32] Harland D, Everitt M J, Nemoto K, Tilma T and Spiller T P 2012 Phys. Rev. A 86062117

[33] Garon A, Zeier R and Glaser S J 2015 Phys. Rev. A 91042122

[34] Koczor B, Zeier R and Glaser S J 2016 Time evolution of coupled spin systems in a generalized Wigner representation (Preprint arXiv:1612.06777v2)

[35] Rundle R P, Mills P W, Tilma T, Samson J H and Everitt M J 2017 Phys. Rev. A 96022117

[36] Leiner D, Zeier R and Glaser S J 2017 Phys. Rev. A 96(6) 063413

[37] Leiner D and Glaser S J 2018 Phys. Rev. A 98(1) 012112

[38] Rundle R P, Tilma T, Samson J H, Dwyer V M, Bishop R F and Everitt M J 2017 A general approach to quantum mechanics as a statistical theory (Preprint arXiv:1708.03814v3)

[39] Newman E T and Penrose R 1966 J. Math. Phys. 7 863-870

[40] Kasperkovitz P 1990 J. Phys. A 235493

[41] Zueco D and Calvo I 2007 J. Phys. A 404635

[42] Agarwal G S and Wolf E 1970 Phys. Rev. D 22187

[43] Moya-Cessa H and Knight P L 1993 Phys. Rev. A 482479

[44] de Gosson M 2017 The Wigner Transform (World Scientific, London)

[45] Agarwal G S and Wolf E 1970 Phys. Rev. D 22161

[46] Groenewold H 1946 Physica 12 405-460

[47] Messiah A 1962 Quantum Mechanics, Vol. II (North-Holland, Amsterdam)

[48] Perelomov A 2012 Generalized Coherent States and Their Applications (Springer, Berlin)

[49] Arecchi F, Courtens E, Gilmore R and Thomas H 1972 Phys. Rev. A 62211

[50] Biedenharn L C and Louck J D 1981 Angular Momentum in Quantum Physics (Addison-Wesley, Reading, MA)

[51] Fano U 1953 Phys. Rev. 90 577-579

[52] Thorne K S 1980 Rev. Mod. Phys. 52299

[53] Seljak U and Zaldarriaga M 1997 Phys. Rev. Lett. 782054

[54] Zaldarriaga M and Seljak U 1997 Phys. Rev. D 551830

[55] Okamoto T and Hu W 2003 Phys. Rev. D 67083002

[56] Reinecke, M and Seljebotn, D S 2013 Astron. Astrophys. 554 A112

[57] Keihänen, E and Reinecke, M 2012 Astron. Astrophys. 548 A110

[58] Wandelt B D and Górski K M 2001 Phys. Rev. D 63(12) 123002

[59] Del Castillo G F T 2012 3-D Spinors, Spin-Weighted Functions and Their Applications (Springer, New York)

[60] Freude D 2006 Quadrupolar Nuclei in Solid-State Nuclear Magnetic Resonance Encyclopedia of Analytical Chemistry ed Meyers R A and Dybowski C doi:10.1002/9780470027318.a6112

[61] Zavatta A, Viciani S and Bellini M 2004 Science 306 660-662 
[62] Zavatta A, Viciani S and Bellini M 2005 Phys. Rev. A 72023820

[63] Zavatta A, Parigi V and Bellini M 2007 Phys. Rev. A 75052106

[64] Barbieri M, Spagnolo N, Genoni M G, Ferreyrol F, Blandino R, Paris M G, Grangier P and Tualle-Brouri R 2010 Phys. Rev. A 82063833

[65] Kumar R, Barrios E, Kupchak C and Lvovsky A 2013 Phys. Rev. Lett. 110130403

[66] Agarwal G and Tara K 1991 Phys. Rev. A 43492

[67] Bopp F 1956 Ann. Inst. H. Poincaré 15 81-112

[68] Leonhardt U and Paul H 1993 Phys. Rev. A 48(6) 4598-4604

[69] Varshalovich D A, Moskalev A N and Khersonskii V K 1988 Quantum Theory of Angular Momentum (World Scientific, Singapore)

[70] Björck Ȧ and Pereyra V 1970 Math. Comp. 24 893-903 